\title{
Observation of high energy atmospheric neutrinos with the Antarctic muon and neutrino detector array
}

J. Ahrens, ${ }^{9}$ E. Andrés, ${ }^{14}$ X. Bai, ${ }^{1}$ G. Barouch,${ }^{11}$ S. W. Barwick, ${ }^{8}$ R. C. Bay, ${ }^{7}$ T. Becka, ${ }^{9}$ K.-H. Becker, ${ }^{2}$ D. Bertrand,${ }^{3}$ F. Binon, ${ }^{3}$ A. Biron, ${ }^{4}$ J. Booth, ${ }^{8}$ O. Botner, ${ }^{13}$ A. Bouchta,,${ }^{4} *$ O. Bouhali, ${ }^{3}$ M. M. Boyce, ${ }^{11}$ S. Carius, ${ }^{5}$ A. Chen, ${ }^{11}$ D. Chirkin,${ }^{7}$ J. Conrad ${ }^{13}$ J. Cooley, ${ }^{11}$ C. G. S. Costa, ${ }^{3}$ D. F. Cowen, ${ }^{10}$ E. Dalberg, ${ }^{14, \uparrow}$ C. De Clercq, ${ }^{15}$ T. DeYoung,,${ }^{11, *}$ P. Desiati, ${ }^{11}$ J.-P. Dewulf, ${ }^{3}$ P. Doksus, ${ }^{11}$ J. Edsjö, ${ }^{14}$ P. Ekström, ${ }^{14}$ T. Feser, ${ }^{9}$ J.-M. Frère, ${ }^{3}$ T. K. Gaisser, ${ }^{1}$ M. Gaug,,${ }^{4}{ }^{8}$ A. Goldschmidt, ${ }^{6}$ A. Hallgren, ${ }^{13}$ F. Halzen, ${ }^{11}$ K. Hanson, ${ }^{10}$ R. Hardtke, ${ }^{11}$ T. Hauschildt, ${ }^{4}$ M. Hellwig, ${ }^{9}$ H. Heukenkamp, ${ }^{4}$ G. C. Hill, ${ }^{11}$ P. O. Hulth, ${ }^{14}$ S. Hundertmark,${ }^{8}$ J. Jacobsen, ${ }^{6}$ A. Karle, ${ }^{11}$ J. Kim,${ }^{8}$ B. Koci, ${ }^{11}$ L. Köpke, ${ }^{9}$ M. Kowalski, ${ }^{4}$ J. I. Lamoureux, ${ }^{6}$ H. Leich, ${ }^{4}$ M. Leuthold ${ }^{4}$ P. Lindahl, ${ }^{5}$ I. Liubarsky, ${ }^{11}$ P. Loaiza, ${ }^{13}$ D. M. Lowder, ${ }^{7, \|}$ J. Madsen, ${ }^{12}$ P. Marciniewski, ${ }^{13, \pi}$ H. S. Matis, ${ }^{6}$ C. P. McParland,${ }^{6}$ T. C. Miller,${ }^{1, * *}$, Y. Minaeva, ${ }^{14}$ P. Miočinović, ${ }^{7}$ P. C. Mock,,${ }^{8, \dagger}$ R. Morse,${ }^{11}$ T. Neunhöffer, ${ }^{9}$ P. Niessen, ${ }^{4,15}$ D. R. Nygren,${ }^{6}$ H. Ögelman,${ }^{11}$ Ph. Olbrechts, ${ }^{15}$ C. Pérez de los Heros, ${ }^{13}$ A. C. Pohl, ${ }^{5}$ R. Porrata,,${ }^{8, \$}$

P. B. Price, ${ }^{7}$ G. T. Przybylski, ${ }^{6}$ K. Rawlins, ${ }^{11}$ C. Reed,${ }^{8, \S}$ W. Rhode,${ }^{2}$ M. Ribordy,${ }^{4}$ S. Richter,,${ }^{11}$ J. Rodríguez Martino, ${ }^{14}$ P. Romenesko, ${ }_{11}$ D. Ross, ${ }^{8}$ H.-G. Sander, ${ }^{9}$ T. Schmidt, ${ }^{4}$ D. Schneider, ${ }^{11}$ R. Schwarz, ${ }^{11}$ A. Silvestri, ${ }^{2,4}$ M. Solarz, ${ }^{7}$ G. M. Spiczak, ${ }^{12}$ C. Spiering, ${ }^{4}$ N. Starinsky, ${ }_{11,\|\|}$ D. Steele, ${ }^{11}$ P. Steffen, ${ }^{4}$ R. G. Stokstad, ${ }^{6}$ O. Streicher, ${ }^{4}$ P. Sudhoff, ${ }^{4}$ K.-H. Sulanke, ${ }^{4}$ I. Taboada, ${ }^{10}$ L. Thollander, ${ }^{14}$ T. Thon, ${ }^{4}$ S. Tilav, ${ }^{1}$ M. Vander Donckt, ${ }^{3}$ C. Walck,${ }^{14}$ C. Weinheimer, ${ }^{9}$ C. H. Wiebusch, ${ }^{4, *}$ C. Wiedeman, ${ }^{14}$ R. Wischnewski, ${ }^{4}$ H. Wissing, ${ }^{4}$ K. Woschnagg, ${ }^{7}$ W. Wu, ${ }^{8}$ G. Yodh, ${ }^{8}$ and S. Young ${ }^{8}$

\author{
(AMANDA Collaboration) \\ ${ }^{1}$ Bartol Research Institute, University of Delaware, Newark, Delaware 19716 \\ ${ }^{2}$ Fachbereich 8 Physik, BUGH Wuppertal, D-42097 Wuppertal, Germany \\ ${ }^{3}$ Université Libre de Bruxelles, Science Faculty CP230, Boulevard du Triomphe, B-1050 Brussels, Belgium \\ ${ }^{4}$ DESY-Zeuthen, D-15735 Zeuthen, Germany \\ ${ }^{5}$ Department of Technology, Kalmar University, S-39182 Kalmar, Sweden \\ ${ }^{6}$ Lawrence Berkeley National Laboratory, Berkeley, California 94720 \\ ${ }^{7}$ Department of Physics, University of California, Berkeley, California 94720 \\ ${ }^{8}$ Department of Physics and Astronomy, University of California, Irvine, California 92697 \\ ${ }^{9}$ Institute of Physics, University of Mainz, Staudinger Weg 7, D-55099 Mainz, Germany \\ ${ }^{10}$ Department of Physics and Astronomy, University of Pennsylvania, Philadelphia, Pennsylvania 19104 \\ ${ }^{11}$ Department of Physics, University of Wisconsin, Madison, Wisconsin 53706 \\ ${ }^{12}$ Physics Department, University of Wisconsin, River Falls, Wisconsin 54022 \\ ${ }^{13}$ Division of High Energy Physics, Uppsala University, S-75121 Uppsala, Sweden \\ ${ }^{14}$ Department of Physics, Stockholm University, SCFAB, SE-10691 Stockholm, Sweden \\ ${ }^{15}$ Vrije Universiteit Brussel, Dienst ELEM, B-1050 Brussel, Belgium
}

(Received 1 February 2002; published 31 July 2002)

\begin{abstract}
The Antarctic muon and neutrino detector array (AMANDA) began collecting data with ten strings in 1997. Results from the first year of operation are presented. Neutrinos coming through the Earth from the Northern Hemisphere are identified by secondary muons moving upward through the array. Cosmic rays in the atmosphere generate a background of downward moving muons, which are about $10^{6}$ times more abundant than the upward moving muons. Over 130 days of exposure, we observed a total of about 300 neutrino events. In the same period, a background of $1.05 \times 10^{9}$ cosmic ray muon events was recorded. The observed neutrino flux is consistent with atmospheric neutrino predictions. Monte Carlo simulations indicate that $90 \%$ of these events lie in the energy range $66 \mathrm{GeV}$ to $3.4 \mathrm{TeV}$. The observation of atmospheric neutrinos consistent with expectations establishes AMANDA-B10 as a working neutrino telescope.
\end{abstract}

DOI: 10.1103/PhysRevD.66.012005 PACS number(s): 95.85.Ry, 95.55.Vj, 96.40.Tv

\footnotetext{
*Now at CERN, CH-1211, Genève 23, Switzerland.

${ }^{\dagger}$ Now at Defense Research Establishment (FOA), S-17290 Stockholm, Sweden.

*Now at Santa Cruz Institute for Particle Physics, University of California—Santa Cruz, Santa Cruz, CA 95064.

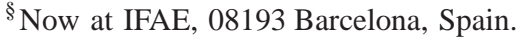

"Now at MontaVista Software, 1237 E. Arques Ave., Sunnyvale, CA 94085.

"Now at The Svedberg Laboratory, S-75121 Uppsala, Sweden.

**Now at Johns Hopkins University, Applied Physics Laboratory, Laurel, MD 20723.

${ }^{\dagger}$ Now at Optical Networks Research, JDS Uniphase, 100 Willowbrook Rd., Freehold, NJ 07728-2879.

\#Now at L-174, Lawrence Livermore National Laboratory, 7000 East Ave., Livermore, CA 94550.

$\S \S$ Now at Dept. of Physics, Massachussetts Institute of Technology, Cambridge, MA.

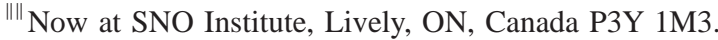




\section{INTRODUCTION}

Energetic cosmic ray particles entering the Earth's atmosphere generate a steady flux of secondary particles such as electrons, muons and neutrinos. The electronic component of cosmic rays is quickly absorbed. High energy muons penetrate the Earth's surface for several kilometers, while atmospheric neutrinos can easily pass the Earth up to very high energies. Interactions of hadronic particles, similar to the ones that create the atmospheric neutrino flux, will generate neutrinos at sites where cosmic rays are generated and where they interact as they travel through the Universe. The goal of observing neutrinos of astrophysical origin determines the design and the size of neutrino telescopes.

The primary channel through which neutrino telescopes detect neutrinos above energies of a few tens of $\mathrm{GeV}$ is by observing the Cherenkov light from secondary muons produced in $\nu_{\mu}$-nucleon interactions in or near the telescope. To ensure that the observed muons are produced by neutrinos, the Earth is used as a filter and only upward moving muons are selected. A neutrino telescope consists of an array of photosensors embedded deeply in a transparent medium. The tracks of high energy muons-which can travel many hundreds of meters, or even kilometers, through water or icecan be reconstructed with reasonable precision even with a coarsely instrumented detector, provided the medium is sufficiently transparent. A location deep below the surface serves to minimize the flux of cosmic-ray muons.

In this paper we demonstrate the observation of atmospheric muon neutrinos with the Antarctic muon and neutrino detector array (AMANDA). These neutrinos constitute a convenient flux of fairly well known strength, angular distribution, and energy spectrum, which can be used to verify the response of the detector. The paper will focus on the methods of data analysis and the comparison of observed data with simulations. After a brief description of the detector, the data and the methods of simulation are introduced in Sec. III and the general methods of event reconstruction are described in Sec. IV. Two AMANDA working groups analyzed the data in parallel. The methods and results of both analyses are described in Secs. V and VI. After a discussion of systematic uncertainties in Sec. VII we present the final results and conclusions.

\section{THE AMANDA DETECTOR}

The AMANDA detector uses the $2.8 \mathrm{~km}$ thick ice sheet at the South Pole as a neutrino target, Cherenkov medium and cosmic ray flux attenuator. The detector consists of vertical strings of optical modules (OMs) - photomultiplier tubes sealed in glass pressure vessels - frozen into the ice at depths of 1500-2000 m below the surface. Figure 1 shows the current configuration of the AMANDA detector. The shallow array, AMANDA-A, was deployed at depths of 800 to 1000 $m$ in 1993-1994 in an exploratory phase of the project. Studies of the optical properties of the ice carried out with AMANDA-A showed a high concentration of air bubbles at these depths, leading to strong scattering of light and making accurate track reconstruction impossible. Therefore, a deeper

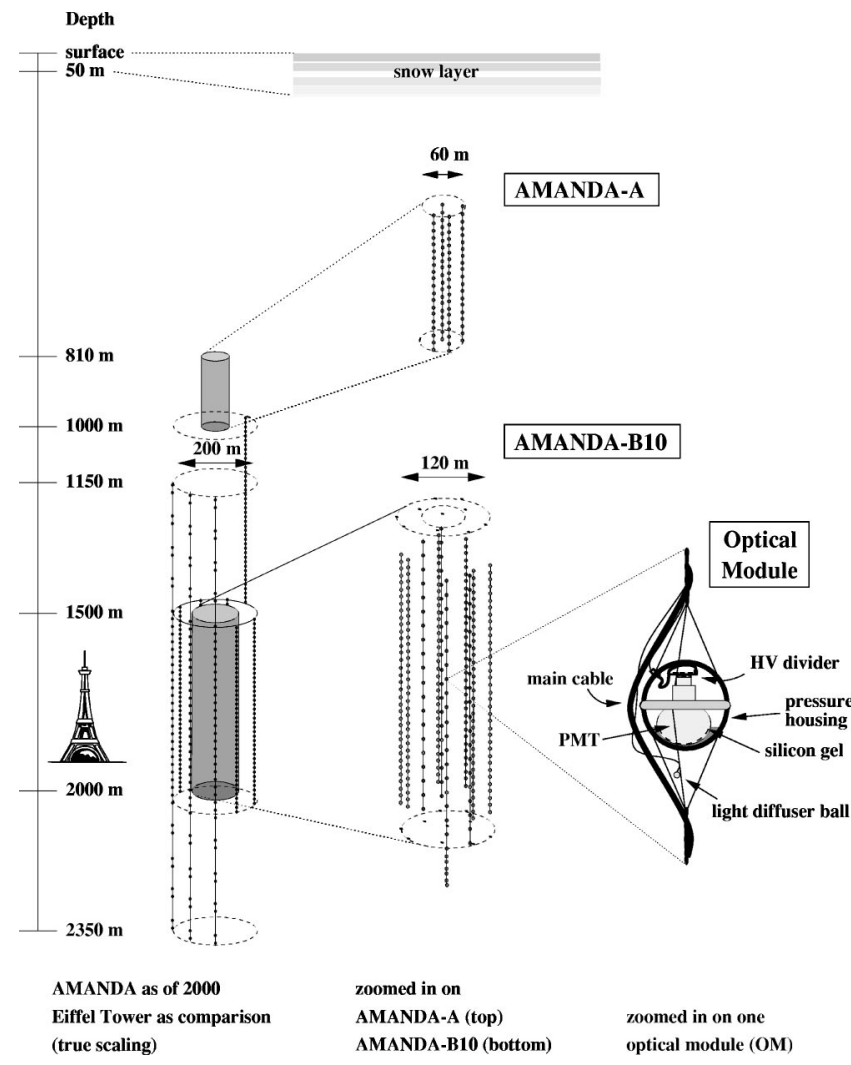

FIG. 1. The present AMANDA detector. This paper describes data taken with the ten inner strings shown in expanded view in the bottom center.

array of ten strings with 302 OMs was deployed in the austral summers of 1995-1996 and 1996-1997 at depths of 1500-2000 m. This detector is referred to as AMANDAB10, and is shown in the center of Fig. 1. The detector was augmented by three additional strings in 1997-1998 and six in 1999-2000, forming the AMANDA-II array.

In AMANDA B10, an optical module consists of a single 8 in. Hamamatsu R5912-2 photomultiplier tube (PMT) housed in a glass pressure vessel. The PMT is optically coupled to the glass housing by a transparent gel. Each module is connected to electronics on the surface by a dedicated electrical cable, which supplies high voltage and carries the anode signal of the PMT. For each event, the optical module is read out by a peak-sensing ADC and a TDC capable of registering up to eight separate pulses. The overall precision of measurement of photon arrival times is approximately 5 ns. Details of deployment, electronics and data acquisition, calibration, and the measurements of geometry, timing resolution, and the optical properties of the ice can be found in $[1,2]$.

The optical properties of the polar ice in which AMANDA is embedded have been studied in detail, using both light emitters located on the strings and the downgoing muon flux itself. These studies [3] have shown that the ice is not perfectly homogeneous, but rather that it can be divided into several horizontal layers which were laid down by varying climatological conditions in the past [4]. Different concentrations of dust in these layers lead to a modulation of the 


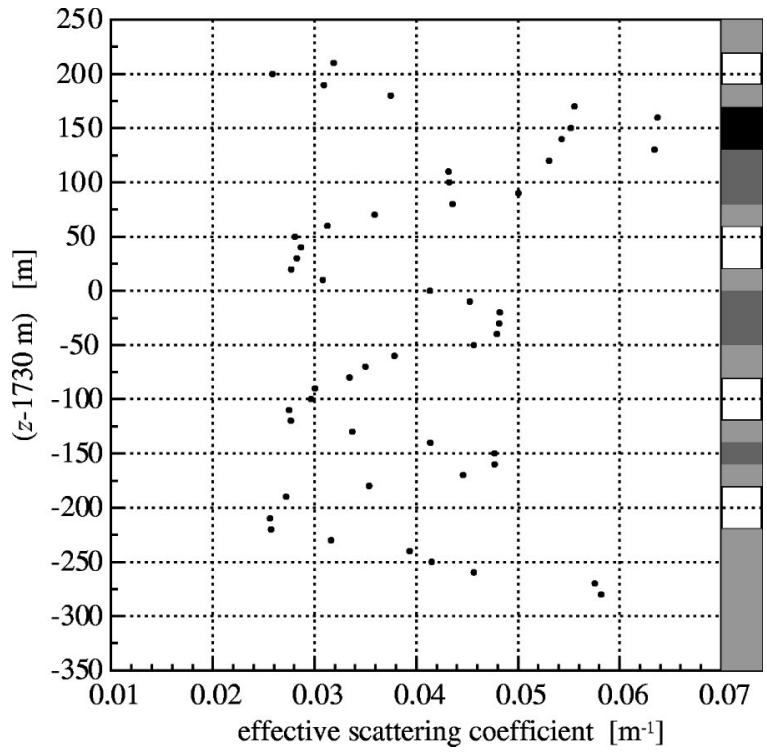

FIG. 2. Variation of the optical properties with depth. The effective scattering coefficient at a wavelength of $532 \mathrm{~nm}$ is shown as a function of depth. The $z$ axis is pointing upwards and denotes the vertical distance from the origin of the detector coordinate system located at a depth of $1730 \mathrm{~m}$. The shaded areas on the side indicate layers of constant scattering coefficient as used in the Monte Carlo simulation.

scattering and absorption lengths of light in the ice, as shown in Fig. 2. The average absorption length is about $110 \mathrm{~m}$ at a wavelength of $400 \mathrm{~nm}$ at the depth of the AMANDA-B10 array, and the average effective scattering length is approximately $20 \mathrm{~m}$.

\section{DATA AND SIMULATION}

The data analyzed in this paper were recorded during the austral winter of 1997, from April to November. Subtracting downtime for detector maintenance, removing runs in which the detector behaved abnormally and correcting for deadtime in the data acquisition system, the effective livetime was 130.1 days.

Triggering was done via a majority logic system, which demanded that 16 or more OMs report signals within a sliding window of $2 \mu \mathrm{s}$. When this condition was met, a trigger veto was imposed and the entire array read out. The raw trigger rate of the array was on average $75 \mathrm{~Hz}$, producing a total data set of $1.05 \times 10^{9}$ events.

Random noise was observed at a rate of $300 \mathrm{~Hz}$ for OMs on the inner four strings and $1.5 \mathrm{kHz}$ for tubes on the outer six, the difference being due to different levels of concentration of radioactive potassium in the pressure vessels (details on noise rates can be found in Ref. [5]). A typical event has a duration of $4.5 \mu \mathrm{s}$, including the muon transit time and the light diffusion times, so random noise contributed on average one PMT signal per event.

Almost all of the events recorded were produced by downgoing muons originating in cosmic ray showers. Triggers from atmospheric neutrinos contribute only a few tens of events per day, a rate small compared to the event rate

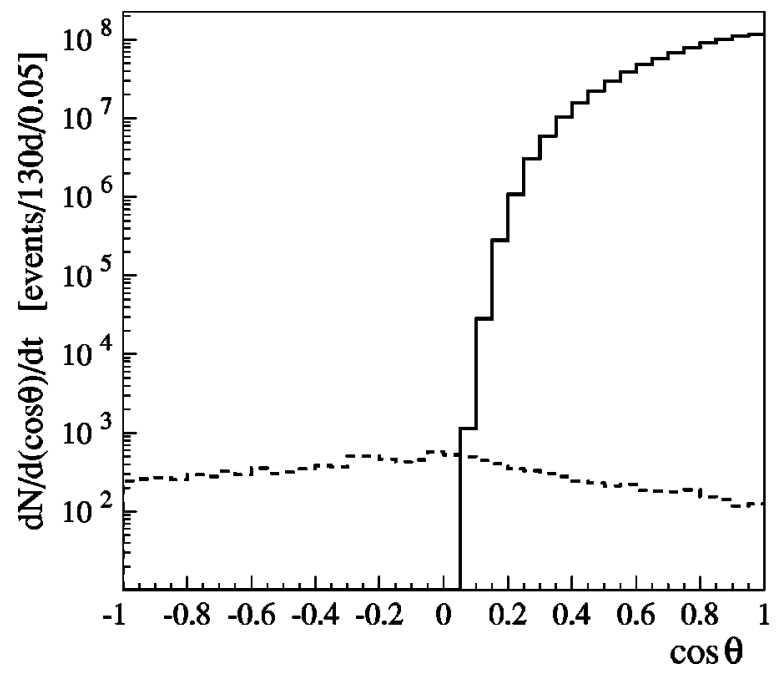

FIG. 3. The zenith angle distribution of simulated AMANDA triggers per 130.1 days of lifetime. The solid line represents triggers from downgoing cosmic ray muons generated by CORSIKA. The dashed line shows triggers produced by atmospheric neutrinos.

from cosmic ray muons, as shown in Fig. 3. The main task of AMANDA data analysis is to separate these neutrino events from the background of cosmic-ray muons. Monte Carlo (MC) simulations of the detector response to muons produced by neutrinos or by cosmic rays were undertaken to develop techniques of background rejection.

Downgoing muons were generated by atmospheric shower simulations of isotropic protons with BASIEV [6] or protons and heavier nuclei with CORSIKA using the QGSJET generator $[7,8]$, and tracked to the detector with the muon propagation code MUDEDX $[9,10]$. Two other muon propagation codes were used to check for systematic differences: PROPMU [11] with a 30\% lower rate and MMC [12] with a slightly higher rate. A total of $0.9 \times 10^{8}$ events were simulated. Most characteristics of the events generated with BASIEV were found to be similar to the more accurate CORSIKA-based simulation. For the latter, the primary cosmic ray flux as described by Wiebel-Sooth and Biermann [13] was used. The curvature of the Earth has been implemented in CORSIKA to correctly describe the muon flux at large zenith angles. The event rate based on this Monte Carlo was 75 $\mathrm{Hz}$ and compares reasonably well with the observed rate of $100 \mathrm{~Hz}$ (after deadtime correction). The detector response to muons was modeled by calculating the photon fields produced by continuous and stochastic muonic energy losses [14], and simulating the response of the hardware to these photons $[15,16]$. Upgoing muons were generated by a propagation of atmospheric neutrinos, which were tracked through the Earth and allowed to interact in the ice in or around the detector or in the bedrock below $[17,18]$. Muons that were generated in the bedrock were propagated using PROPMU [11] until they reached the rock-ice boundary at the depth of 2800 $\mathrm{m}$. The muons were then propagated through the ice in the same way as those from cosmic ray showers. The atmospheric neutrino flux was taken from Lipari [19].

The Cherenkov photon propagation through the ice was modeled to create multidimensional tables of density and 
arrival time probability distributions of the photon flux. These photon fields were calculated for pure muon tracks and for cascades of charged particles. A real muon track was modeled as a superposition of the photon fields of a pure muon track and the stochastic energy losses based on cascades. The photon fields were calculated out to $400 \mathrm{~m}$ from the emission point, taking into account the orientation of the OM with respect to the muon or cascade. In the detector simulation, the ice was modeled as 16 discrete layers, as indicated by the shaded areas in Fig. 2. The spectral properties of the photomultiplier sensitivity, the glass, the gel, and, most importantly, the ice itself were included in the simulation of the photon propagation. The probability of photon detection depends on the Fresnel reflectance at all interfaces, transmittances of various parts, and quantum and collection efficiencies of the PMT. The relevant physical parameters have been measured in the laboratory, so that the spectral sensitivity of the OM could be evaluated. Two types of OMs, differing in the type of pressure vessel, were used in the construction of AMANDA-B10. The inner four strings (AMANDA-B4) use Billings housings while the outer six strings use Benthos housings. (Benthos Inc. and Billings Industries are the manufacturers of the glass pressure vessels. Benthos and Billings are registered trademarks of the respective companies.) The two types of housing have different optical properties. The Benthos OMs have an effective quantum efficiency of $21 \%$ at a wavelength of $395 \mathrm{~nm}$ for planewave photons incident normal to the PMT photocathode. Ninety percent of the detected photons are in the spectral range of $345-560 \mathrm{~nm}$.

An additional sensitivity effect arises from the ice surrounding the OMs. The deployment of OMs requires melting and refreezing of columns of ice, called "hole ice" hereafter. This cycle results in the formation of bubbles in the vicinity of the modules, which increase scattering and affects the sensitivity of the optical modules in ways that are not understood in detail. Since the total volume of hole ice is small compared to bulk ice in the detector (columns of $60 \mathrm{~cm}$ diameter, compared to $30 \mathrm{~m}$ spacing between strings), its effect on optical properties can be treated as a correction to the OM angular sensitivity. The increased scattering of photons in the hole ice has been simulated and compared to data taken with laser measurements in situ to assess the magnitude of this effect. This comparison provides an OM sensitivity correction that reduces the relative efficiency in the forward direction, but enhances it in the sideways and backward directions. The sensitivity in the backward hemisphere $\left(90^{\circ}-180^{\circ}\right)$ relative to the sensitivity integrated over all angles $\left(0^{\circ}-180^{\circ}\right)$ of the optical sensor increases from $20 \%$ to $27 \%$, due to this correction, while the average relative sensitivity in the forward direction $\left(0^{\circ}-90^{\circ}\right)$ drops from $80 \%$ to $73 \%$. In other words, an OM becomes a somewhat more isotropic sensor.

The effective angular sensitivity of the OMs was also assessed using the flux of downgoing atmospheric muons as a test beam illuminating both the 295 downward facing OMs and the 7 upward facing OMs. We assumed that the response of the upward facing OMs to light from downward muons is equivalent to the response of the downward facing OMs to light from upward moving muons. Based on this assumption we derived a modified angular response function (later referred to as ANGSENS), which resulted in a effective reduction of the absolute OM sensitivity in forward direction. In this model the effective relative sensitivity is $67 \%$ in the forward hemisphere, and 33\% in the backward hemisphere. This correction will be used to estimate the effect of systematic uncertainties in the angular response on the final neutrino analysis.

The simulation of the hardware response included the modeling of gains and thresholds and random noise at the levels measured for each OM. The transit times of the cables and the shapes of the photomultiplier pulses, ranging from 170 to 360 ns full width at half maximum (FWHM), were included in the trigger simulation. Multi-photon pulses were simulated as superimposed single photoelectron waveforms. In all, some $8 \times 10^{5}$ seconds of cosmic rays were simulated, corresponding to $7 \%$ of the events contained in the 1997 data set.

\section{EVENT RECONSTRUCTION}

The reconstruction of muon events in AMANDA is done offline, in several stages. First, the data are "cleaned" by removing unstable PMTs and spurious PMT signals (or "hits") due to electronic or PMT noise. The cleaned events are then passed through a fast filtering algorithm, which reduces the background of downgoing muons by one order of magnitude. This reduction allows the application of more sophisticated reconstruction algorithms to the remaining data set.

Because of the complexity of the task, and in order to increase the robustness of the results, two separate analyses of the 1997 data set were undertaken. Both proceeded along the general lines described above, but differ in the details of implementation. The preliminary stages, which are very similar in both analyses, are described here. The particulars of each analysis will be described in Secs. V and VI. A more detailed description of the reconstruction procedure will be published elsewhere [20].

\section{A. Cleaning and filtering}

The first step in reconstructing events is to clean and calibrate the data recorded by the detector. Unstable channels (OMs) are identified and removed on a run-to-run basis. On average, 260 of the 302 OMs deployed are used in the analyses. The recorded times of the hits are corrected for delays in the cables leading from the OMs to the surface electronics and for the amplitude-dependent time required for a pulse to cross the discriminator threshold. Hits are removed from the event if they are identified as being due to instrumental noise, either by their low amplitudes or short pulse lengths, or because they are isolated in space by more than $80 \mathrm{~m}$ and time by more than $500 \mathrm{~ns}$ from the other hits recorded in the event. Pulses with short duration, measured as the time over threshold (TOT), are often related to electronic cross-talk in the signal cables or the surface electronics. In analysis II, TOT cuts are applied to individual channels beyond the standard cleaning common to both analyses (see Sec. VI). 
Following the cleaning and the calibration, a "line fit" is calculated for each event. This fit is a simple $\chi^{2}$ minimization of the apparent photon flux direction, for which an analytic solution can be calculated quickly [21] (see also [1]). It contains no details of Cherenkov radiation or propagation of light in the ice. Hits arriving at time $t_{\mathrm{i}}$ at PMT $i$ located at $\vec{r}_{i}$ are projected onto a line. The minimization of $\chi^{2}=\Sigma_{i}\left(\vec{r}_{i}\right.$ $\left.-\vec{r}_{0}-\vec{v}_{\text {lf }} \cdot t_{i}\right)^{2}$ gives a solution for $\vec{r}_{0}$ and a velocity $\vec{v}_{\text {lf }}$. The results of this fit-at the first stage the direction $\vec{v}_{\text {lf }} /\left|\vec{v}_{\text {lf }}\right|$, at later stages the absolute value of the velocity-are used to filter the data set. Approximately $80-90 \%$ of the data, for which the line fit solution is steeply downgoing, are rejected at this stage.

\section{B. Maximum likelihood reconstruction}

After the data have been passed through the fast filter, tracks are reconstructed using a maximum likelihood method. The observed photon arrival times do not follow a simple Gaussian distribution attributable to electronic jitter; instead, a tail of delayed photons is observed. The photons can be delayed predominantly by scattering in the ice that causes them to travel on paths longer than the length of the straight line inclined at the Cherenkov angle to the track. Also, photons emitted by scattered secondary electrons generated along the track will have emission angles other than the muon Cherenkov angle. These effects generate a distribution of arrival times with a long tail of delayed photons.

We construct a probability distribution function describing the expected distribution of arrival times, and calculate the likelihood $\mathcal{L}_{\text {time }}$ of a given reconstruction hypothesis as the product of the probabilities of the observed arrival times in each hit OM:

$$
\mathcal{L}_{\text {time }}=\prod_{i=1}^{N_{\text {hit }}} p\left(t_{\text {res }}^{(i)} \mid d_{\perp}^{(i)}, \theta_{\text {ori }}^{(i)}\right)
$$

where $t_{\text {res }}=t_{\text {obs }}-t_{\text {Cher }}$ is the time residual (the delay of the observed hit time relative to that expected for unscattered propagation of Cherenkov photons emitted by the muon), and $d_{\perp}$ and $\theta_{\text {ori }}$ are the distance of the OM from the track and the orientation of the module with respect to the track. The probability distribution function $p$ includes the effects of scattering and absorption in the bulk ice and in the refrozen ice around the modules. The functional form of $p$ is based on a solution to a transport equation of the photon flux from a monochromatic point source in a scattering medium $[22,23]$. The free parameters of this function are then fit to the expected time profiles that are obtained by a simulation of the photon propagation from muons in the ice [14,22]. Varying the track parameters of the reconstruction hypothesis, we find the maximum of the likelihood function, corresponding to the best track fit for the event. The result of the fit is described by five parameters: three $(x, y, z)$ to determine a reference point, and two $(\theta, \phi)$ for the zenith and azimuth of the track direction. Figure 4 shows an event display of two upgoing muon events together with the reconstructed tracks.

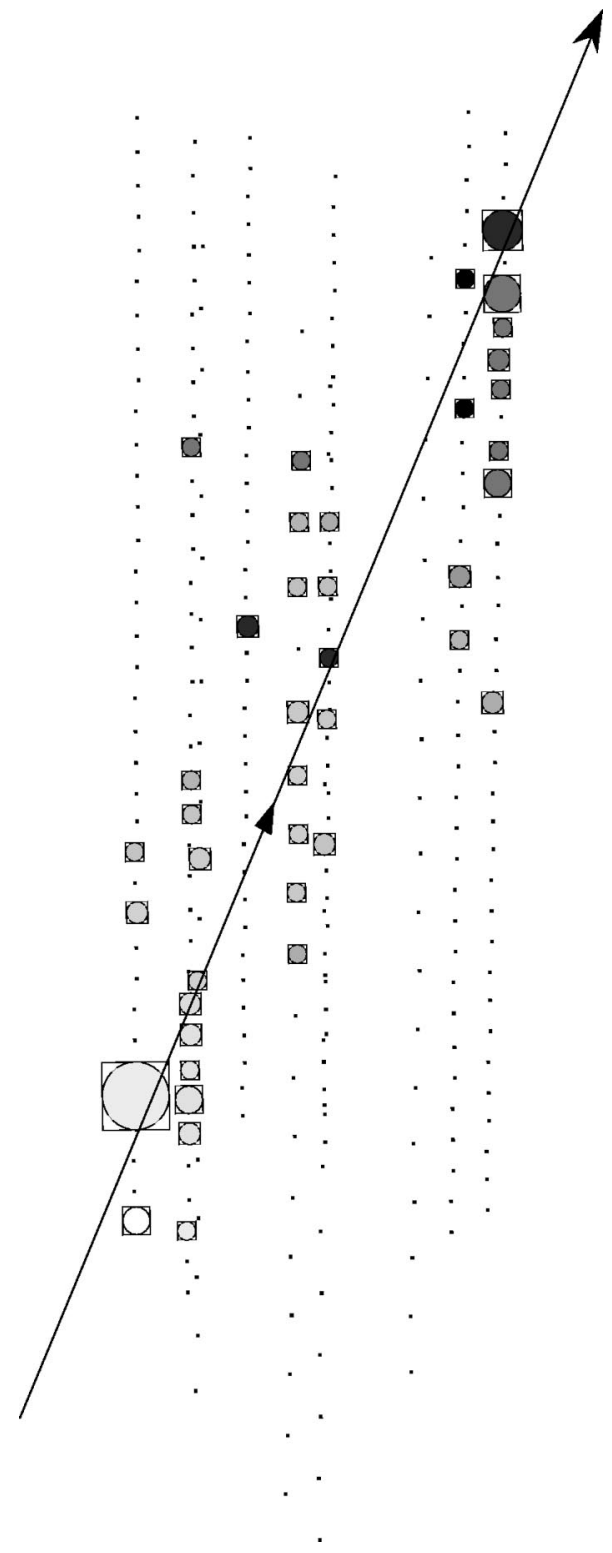

FIG. 4. Event display of an upgoing muon event. The gray scale indicates the flow of time, with early hits at the bottom and the latest hits at the top of the array. The arrival times match the speed of light. The sizes of the circles correspond to the measured amplitudes.

\section{Quality parameters}

The set of apparently upgoing tracks provided by the reconstruction procedure exceeds the expected number of upgoing tracks from atmospheric neutrino interactions by one to three orders of magnitude, depending on the details of the reconstruction algorithm (see Secs. V and VI). In order to reject the large number of "fake events"—events generated by a downgoing muon or cascade, but seemingly having an upgoing structure-we impose additional requirements on the reconstructed events to obtain a relatively pure neutrino sample. These requirements consist of cuts on observables derived from the reconstruction and on topological event parameters. Below, we describe the most relevant of the parameters used. 


\section{Reduced likelihood, $L$}

In analogy to a reduced $\chi^{2}$, we define a reduced likelihood

$$
L=\frac{-\ln \mathcal{L}_{\text {time }}}{N_{\text {hit }}-5}
$$

where $N_{\text {hit }}-5$, the number of recorded hits in the event less the five track fit parameters, is the number of degrees of freedom. A smaller $L$ corresponds to a higher quality of the fit.

\section{Number of direct hits, $N_{\text {dir }}$}

The number of direct hits is defined as the number of hits with time delays $t_{\text {res }}$ smaller than a certain value. We use time intervals of $[-15 \mathrm{~ns},+25 \mathrm{~ns}]$ and $[-15 \mathrm{~ns}$, $+75 \mathrm{~ns}]$, and denote the corresponding parameters as $N_{\mathrm{dir}}^{(25)}$ and $N_{\text {dir }}^{(75)}$, respectively. The negative extent of the window allows for jitter in PMT rise times and for small errors in geometry and calibration, while the positive side includes these effects as well as delays due to scattering of the photons. Events with many direct hits (i.e., only slightly delayed photons) are likely to be well reconstructed.

\section{Track length, $L_{d i r}$}

The track length is defined by projecting each of the direct hits onto the reconstructed track, and measuring the distance between the first and the last hit. A cut on this parameter rejects events with a small lever arm for the reconstruction. Direct hits with time residuals of $[-15 \mathrm{~ns},+75 \mathrm{~ns}]$ are used for the measurement of the track length. Cuts on the absolute length, as well as zenith angle dependent cuts (which take into account the cylindrical shape of the detector) have been used. The requirement of a minimum track length corresponds to imposing a muon energy threshold. For example, a track length of $100 \mathrm{~m}$ translates into a muon energy threshold of about $25 \mathrm{GeV}$.

\section{Smoothness, $S$}

The "smoothness" parameter is a check on the selfconsistency of the fitted track. It measures the constancy of light output along the track. Highly variable apparent emission of light usually indicates that the track either has been completely misreconstructed or that an underlaying muonic Cherenkov light was obscured by a very bright stochastic light emission, which usually leads to poor reconstruction. The smoothness parameter was inspired by the KolmogorovSmirnov test of the consistency of two distributions; in our case the consistency of the observed hit pattern with the hypothesis of constant light emission by a muon.

Figure 5 shows two events to illustrate the characteristics of the smoothness parameter. One event is a long uniform track, which was well reconstructed. The other event is a background event which displays a very poor smoothness.

The simplest definition of the smoothness is given by

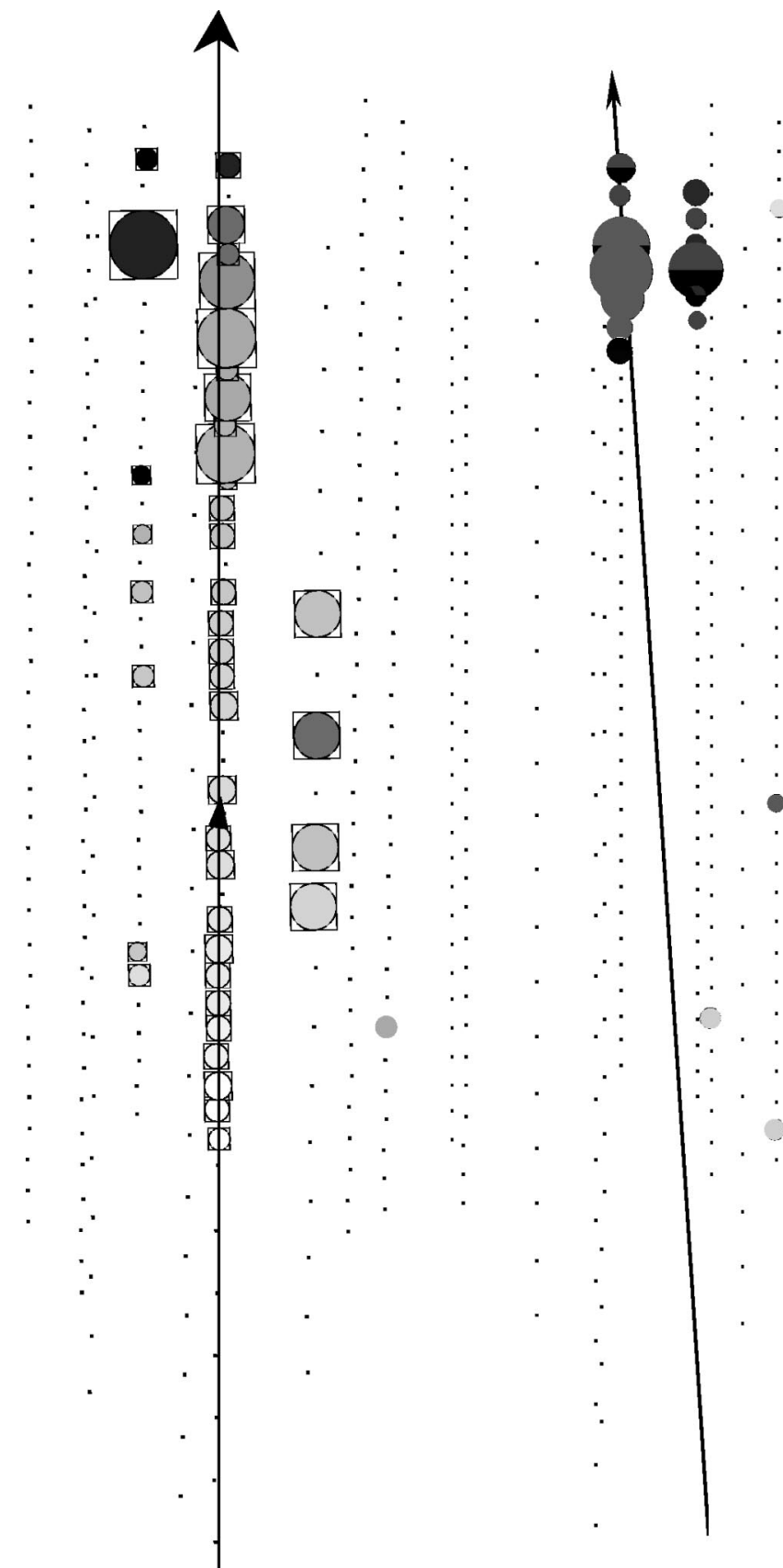

(a)

(b)

FIG. 5. Two muon events: The upgoing muon event shown on the left has a smooth distribution of hits along the track. The tracklike hit topology of this event can be used to distinguish it from background events. The event on the right is a background event with a poor smoothness value.

$$
S=\max \left(\left|S_{j}\right|\right), \quad \text { where } \quad S_{j}=\frac{j-1}{N-1}-\frac{l_{j}}{l_{N}} .
$$

Figure 6 illustrates the smoothness parameter for the two events displayed in Fig. 5. Here $l_{j}$ is the distance along the track between the points of closest approach of the track to the first and the $j^{\text {th }}$ hit modules, with the hits taken in order of their projected position on the track. $N$ is the total number 


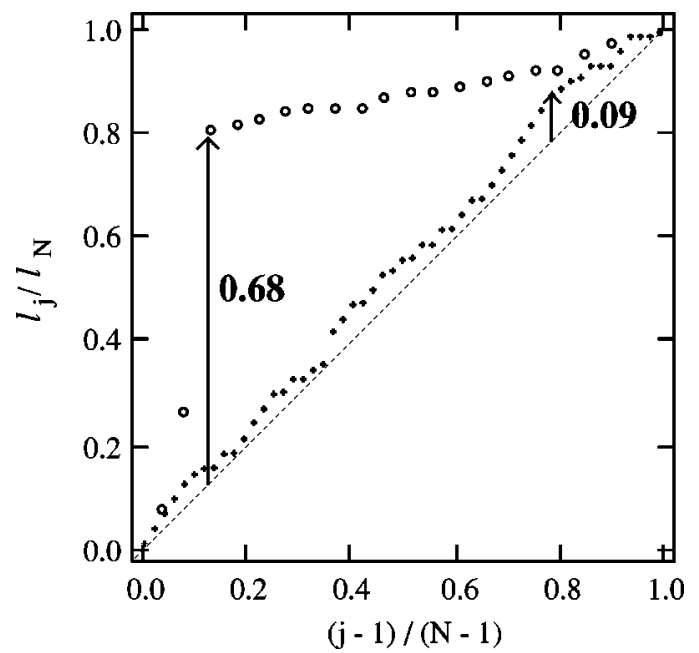

FIG. 6. Illustration of the smoothness parameter, which compares the observed distribution of hits to that predicted for a muon emitting Cherenkov light. In the simplest formulation, shown here, the prediction is given as a straight line. A large deviation from a straight line (0.68) is found for the event on the right in Fig. 5. The high quality track-like event on the left in Fig. 5 displays a small deviation (0.09).

of hits. Tracks with hits clustered at the beginning or end of the track have $S_{j}$ approaching +1 or -1 , leading to $S=1$. High quality tracks such as the event on the left side of Fig. 5 , with $S$ close to zero, have hits equally spaced along the track.

\section{Sphericity}

Treating the hit modules as point masses, we can form a tensor of inertia for each event, describing the spatial distribution of the hits. Diagonalizing the tensor of inertia yields as eigenvalues $I_{i}$ the moments of inertia about the principal axes of rotation. For a long, cylindrical distribution of hit modules, two moments will be much larger than the third. We can reject spherical events, such as those produced by muon bremsstrahlung, by requiring that the normalized magnitude of the smallest moment, $I_{1} / \Sigma I_{i}$, be small.

\section{Principal methods of the analyses}

The two analyses of the data diverge after the filtering stage, following different approaches to event reconstruction and background rejection.

Analysis I uses an improved likelihood function based on a more detailed description of the photon response [22], followed by a set of stepwise tightened cuts. Analysis II uses a Bayesian reconstruction [24] in which the likelihood is multiplied by a zenith angle dependent prior function, resulting in a strong rejection of downgoing background.

Rare backgrounds due to unsimulated instrumental effects, such as cross-talk between signal channels and unstable voltage supply, were identified in the course of the analyses. These effects either produced spurious triggers, or, more often, spurious hits that caused the event to be misreconstructed. Different but comparably efficient techniques were developed to treat these backgrounds. In analysis I the event topology is inspected; if the spatial pattern of hit OMs is inconsistent with the reconstructed muon trajectory, the event is rejected. Analysis II attempts to remove the anomalous hits or triggers through identification of characteristic correlations in signal amplitudes and times, which considerably reduces the rate of these misreconstructions.

At this stage the data set in each analysis is reduced to several thousand events out of the original $1.05 \times 10^{9}$, but the data are still background dominated. The prediction for atmospheric neutrinos is about 500 at this point.

For the final selection of a nearly pure sample of neutrino induced events, cuts on characteristic observables are tightened until the remaining background disappears. The two analyses use different techniques to choose their final cuts, but obtain comparable efficiencies. Further details of the analyses can be found in Refs. [25-27].

\section{ANALYSIS I}

In this analysis the data were processed through three levels of initial cuts, designed to reduce the number of background events to a manageable size for the final cut evaluation. After a first filtering based on the line fit (level 1), cuts on the zenith angle, the number of direct photons, and the likelihood of the fitted track obtained by the maximum likelihood reconstruction were applied (level 2).

\section{A. Removal of cascade-like events and detector artifacts}

A third filter level used the results of an iterative likelihood reconstruction with varying track initializations, a fit based on the hit probabilities [see Eq. (4)] and a reconstruction to the hypothesis of a high energy cascade, e.g., due to a bright seconday muon bremsstrahlung interaction.

The first two levels of filtering consisted of relatively weak cuts on basic parameters like the zenith angle and likelihood. They reduced the data set to about $4 \times 10^{5}$ events. At this stage, residual unsimulated instrumental features become apparent, e.g., comparatively high amplitude cross-talk produced when a downgoing muon emits a bright shower in the center of the detector. Such events are predominantly reconstructed as moving vertically upward and can be identified in the distribution of the center of gravity (COG) of hits. Its vertical component $\left(z_{\mathrm{COG}}\right)$ shows unpredicted peaks in the middle and the bottom of the detector [see also Fig. 14 (top), demonstrating the effect for analysis II], while the horizontal components $\left(x_{\mathrm{COG}}\right.$ and $\left.y_{\mathrm{COG}}\right)$ show an enhancement of hits towards the outer strings. These strings are read out via twisted pair cables, as opposed to the coaxial cables used on the inner strings. The twisted pair cables were found to be more susceptible to cross-talk signals. Note that variations in the optical parameters of the ice due to past climatological episodes also produce some vertical structure.

We developed additional COG cuts on the topology of the events in order to remove these backgrounds. These cuts, which depend on the reconstructed zenith angle, use the track lengths $L_{\text {dir }}$ and the normalized smallest eigenvalues of the tensor of inertia $\left(I_{1} / \Sigma I_{i}\right)$. 

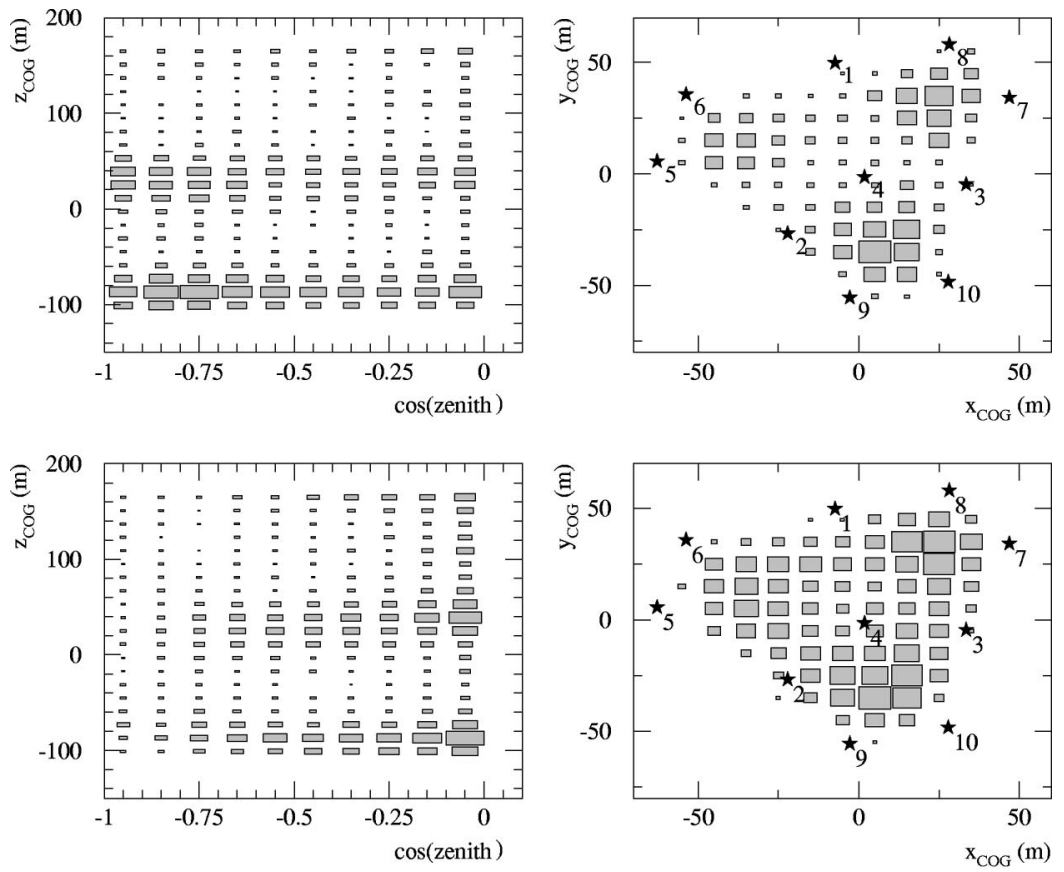

FIG. 7. Characteristic distributions of the center of gravity (COG) of events. The figures on the left show the distribution of the depth $z_{\mathrm{COG}}$ versus the reconstructed zenith angle. The figures on the right show the horizontal location of events in the $x_{\mathrm{COG}^{-}} y_{\mathrm{COG}}$ plane of events with $0 \mathrm{~m}<z_{\mathrm{COG}}$ $<50 \mathrm{~m}$. The positions of the strings are marked by stars. Top: Experimental data before application of the COG cuts. Middle: Experimental data after application of the COG cuts. Bottom: Expectation from the BG simulation after cuts.

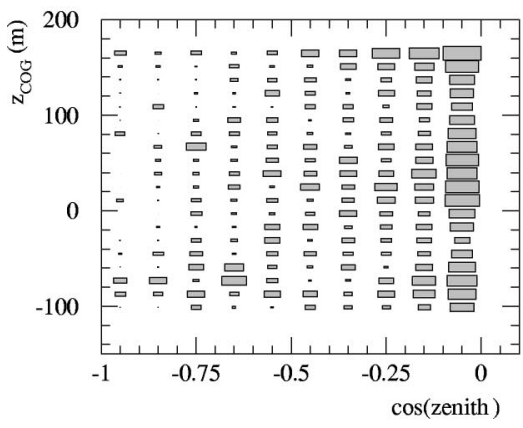

Figure 7 shows the different components of the center of gravity of the hits and the reconstructed zenith angle before and after application of the COG cuts, and the Monte Carlo prediction for fake upward events stemming from misreconstructed downgoing muons. The cuts remove most of the unsimulated background-in particular that far from the horizon-and bring experiment and simulation into much better agreement.

In order to verify the signal passing rates, these cuts and those from the previous levels were applied to a subsample of unfiltered (i.e., downgoing) events but with the zenith angle dependence of the cuts reversed, thus using the abundant cosmic ray muons as stand-ins for upgoing muons.

In all, these three levels of filtering reduced the data set by a factor of approximately $10^{5}$ (see Table II).

\section{B. Multi-photoelectron likelihood and hit likelihood}

Before the final cut optimization the last, most elaborate reconstruction was applied, combining the likelihoods for the arrival time of the first of muliple photons in a PMT with the likelihoods for PMTs to have been hit or have not been hit.

The probability densities $p\left(t_{\text {res }}^{(i)} \mid d_{\perp}^{(i)}, \theta_{\text {ori }}^{(i)}\right)$ [see Eq. (1), Sec. IV B] describe only the arrival times of single photons. Density functions for the multi-photoelectron case have to include the effect of repeatedly sampling the distribution of photon arrival times. For several detected photons, the first of them is usually less scattered than the average photon (which defines the single photoelectron case). Therefore the leading edge of a PMT pulse composed of multiple photoelectrons (MPE) will be systematically shifted to earlier times compared to a single photoelectron. The MPE likelihood $\mathcal{L}_{\text {time }}^{\mathrm{MPE}}$ [22] uses the recorded amplitude information to model this shift.

In the reconstructions mentioned so far, the timing information from hit PMTs was used. However, a PMT which was not hit also delivers information. The hit likelihood $\mathcal{L}_{\text {hit }}$ does not depend on the arrival times but represents the probability that the track produced the observed hit pattern. It is constructed from the probability densities $p_{\text {hit }}\left(d_{\perp}^{(i)}, \theta_{\text {ori }}^{(i)}\right)$ that a given PMT $i$ was hit if it was in fact hit, and the probabilities $\left[1-p_{\text {hit }}\left(d_{\perp}^{(j)}, \theta_{\text {ori }}^{(j)}\right)\right]$ that a given PMT $j$ was not hit if it was not hit:

$$
\mathcal{L}_{\text {hit }}=\prod_{i=1}^{N_{\text {hit }}} p_{\text {hit }}\left(d_{\perp}^{(i)}, \theta_{\text {ori }}^{(i)}\right) \prod_{i=N_{\text {hit }}+1}^{N_{\mathrm{OM}}}\left(1-p_{\text {hit }}\left(d_{\perp}^{(i)}, \theta_{\text {ori }}^{(i)}\right)\right)
$$

where the first product runs over all hit PMTs and the second over all non-hit PMTs.

The likelihood combining these two probabilities is 


$$
\mathcal{L}=\mathcal{L}_{\text {time }}^{\mathrm{MPE}} \cdot \mathcal{L}_{\text {hit }}
$$

A cut on the reconstructed zenith angle obtained from fitting with $\mathcal{L}$ leaves less than $10^{4}$ events in the data set, defined as level 4 in Fig. 8.

\section{Final separation of the neutrino sample}

For the final stage of filtering, a method (CUTEVAL) was developed to select and optimize the cuts taking into account correlations between the cut parameters. A detailed description of this method can be found in [27]. The principle of CUTEVAL is to numerically optimize the ratio of signal to $\sqrt{\text { background }}$ by variation of the selection of cut parameters, as well as the actual cut values. Parameters are used only if they improve the efficiency of separation over optimized cuts on all other already included parameters. A first optimization was based purely on Monte Carlo simulations, with simulated atmospheric neutrinos for signal and simulated downgoing muons forming the background. This optimization yielded four such independent parameters. Two other optimizations involved experimental data. In both cases, experimental data have been defined as the background sample. In one case, the signal was represented by atmospheric neutrino Monte Carlo simulations, in the other by experimental data subjected to zenith angle inverted cuts (i.e., to downward events passing the quality cuts, but being "good" events with respect to the upper hemisphere instead-like neutrino candidates-with respect to the lower hemisphere). These latter optimizations yielded two additional parameters, which rejected a small contribution of residual unsimulated backgrounds: coincident muons from simultaneous independent air showers and events accompanied by instrumental artifacts such as cross-talk. After application of these two cuts to simulated and experimental data, the distributions of observables agree to a satisfactory precision.

Once the minimal set of parameters is found, the optimal cut values can be represented as a function of the number of background events $N_{\mathrm{BG}}$ passing the cuts. The result is a path through the cut parameter space which yields the best signal efficiency for any desired purity of the signal, characterized by $N_{\mathrm{BG}}$. Using this representation, one can calculate the number of events passing the cuts as a function of the fitted $N_{\mathrm{BG}}$ for signal and for background Monte Carlo program. Figure 8 (top) shows this dependence for simulations as well as for experimental data, with $N_{\mathrm{BG}}$ varying from trigger level to a level that leaves only a few events in the data set. One observes that the actual background expectation falls roughly linearly as the fitted $N_{\mathrm{BG}}$ is reduced. Below values of a few hundred events the signal is expected to dominate the event sample. The experimental curve follows the expectation from the sum of background and signal Monte Carlo program. For large $N_{\mathrm{BG}}$, the observed event rate follows the background expectation. At smaller $N_{\mathrm{BG}}$, the experimental shape turns over into the signal expectation and follows it nicely down to the sample of events with highest quality (the smallest values of $N_{\mathrm{BG}}$ ). For a moderate background contamination of $N_{\mathrm{BG}}$ $=10$, one gets a total of 223 neutrino candidates. The param-
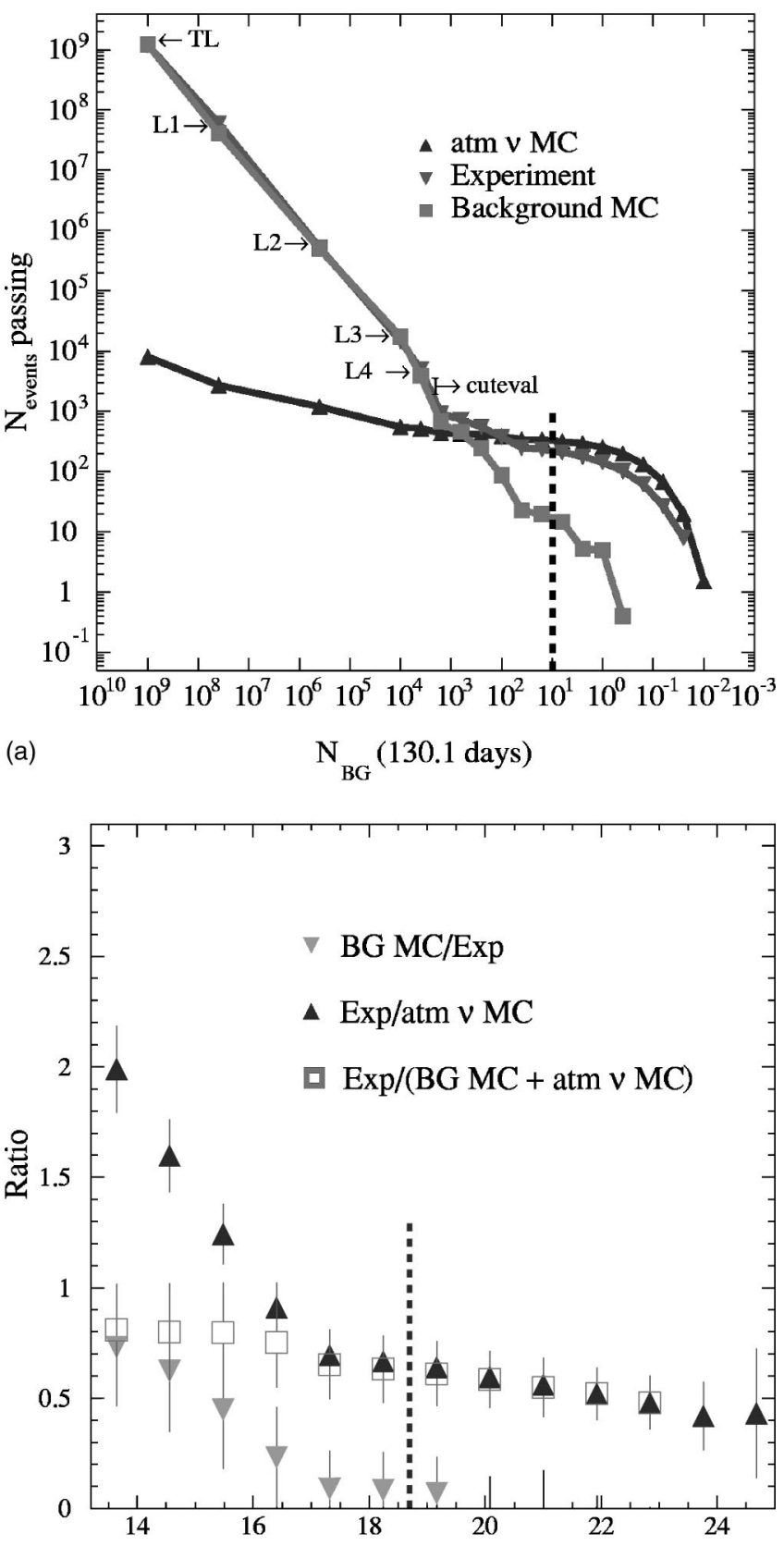

(b)

Event quality $\mathrm{Q}=\ln \left(\mathrm{N}_{0} / \mathrm{N}_{\mathrm{BG}}\right)$

FIG. 8. The fitted background parameter $N_{\mathrm{BG}}$. Top: Number of events versus $N_{\mathrm{BG}}$. Smaller values of $N_{\mathrm{BG}}$ correspond to harder cuts. Below $N_{\mathrm{BG}}=1500$ the CUTEVAL parametrization was used to calculate the cut values corresponding to $N_{\mathrm{BG}}$. For larger values of $N_{\mathrm{BG}}$ the data points correspond to the cuts from the filter levels: Level 4 (see Sec. V B), level 3, level 2, level 1, and trigger level (Table II). Bottom: Ratios of events passing in the experimental data compared to various Monte Carlo expectations for signal and background as a function of event quality. The dashed line indicates the final cuts.

eters and cut values as obtained by the CUTEVAL procedure are summarized in Table I.

Figure 8 (bottom) translates the background parameter $N_{\mathrm{BG}}$ into an event quality parameter $Q$, defined as $Q$ $\equiv \ln \left(N_{0} / N_{\mathrm{BG}}\right)=\ln \left(1.05 \cdot 10^{9} / N_{\mathrm{BG}}\right)$. The plot shows the ratios 
TABLE I. Final quality parameters and cuts obtained from the cut evaluation procedure. The "direct" time interval for variables $N_{\text {dir }}, L_{\text {dir }}$, and $S_{\text {dir }}$ is $[-15 \mathrm{~ns},+75 \mathrm{~ns}]$. The first four rows show cut parameters obtained by all (Monte Carlo and experimental) searches; the last two rows show two additional (weaker) cuts, which were found to remove unsimulated backgrounds.

\begin{tabular}{|c|c|c|}
\hline Parameter & Cut & Explanation \\
\hline$|S|$ & $<0.28$ & See Sec. IV C 4 \\
\hline$\left|S^{P_{\mathrm{hit}}}\right| /\left(\theta_{\mathrm{mpe}}-90^{\circ}\right)$ & $<0.01$ & $\begin{array}{l}\text { Tightens the requirement on the smoothness for } \\
\text { tracks }\end{array}$ \\
\hline & & close to the horizon where background is high \\
\hline$\left(N_{\mathrm{dir}}-2\right) \cdot L_{\mathrm{dir}}$ & $>750 \mathrm{~m}$ & $\begin{array}{c}\text { Lever arm of the track times the number of } \\
\text { supporting points }\end{array}$ \\
\hline $\log \left(\mathcal{L}_{\text {up }} / \mathcal{L}_{\text {down }}\right)$ & $<-7.7$ & $\begin{array}{l}\text { Ratio of the likelihoods of the best } \\
\text { upgoing and best downgoing hypotheses }\end{array}$ \\
\hline \multirow[t]{2}{*}{$\Psi$ (mpe,lf) } & $<35^{\circ}$ & $\begin{array}{l}\text { Space angle between the results from the } \\
\text { multi-photon }\end{array}$ \\
\hline & & $\begin{array}{l}\text { likelihood reconstruction and the line fit. This cut } \\
\text { effectively removes cross-talk features. }\end{array}$ \\
\hline$\sqrt{\left(S_{\mathrm{dir}}\right)^{2}+\left(S_{\mathrm{dir}}^{P_{\mathrm{hit}}}\right)^{2}}$ & $<0.55$ & $\begin{array}{l}\text { Parameter combining the two smoothness } \\
\text { definitions (here calculated using only direct hits) } \\
\text { This cut effectively removes coincident muon } \\
\text { events from independent air showers. }\end{array}$ \\
\hline
\end{tabular}

of events from the upper figure as a function of $Q$. At higher qualities $(Q>17)$, the ratio of observed events to the atmospheric neutrino simulation flattens out with a further variation of only $30 \%$. The value at $Q=17$ is approximately 0.6 for the standard Monte Carlo program (chosen in Fig. 8, top) and approximately unity for the ANGSENS Monte Carlo program (chosen in Fig. 8, bottom).

Table II lists the cut efficiencies for the atmospheric neutrino simulation (with and without the implementation of the angular sensitivity fitted model ANGSENS of the OMs-see Secs. III and VII), the background simulation of atmospheric muons from air showers (without ANGSENS) and the experimental data. Again, the experimental numbers agree well with the background simulation up to the first two filter levels. Later, the Monte Carlo program underestimates the experimental passing rates slightly. The last row shows the expected numbers of events for the last stage of filtering. If, in addition, the effect of neutrino oscillations (see Sec. VII) is included, the atmospheric neutrino simulation including the ANGSENS model predicts 224 events, in closest agreement with the experiment. However, the $5 \%$ effect due to oscillations is smaller than our systematic uncertainty (see Sec. VII).

\section{Characteristics of the neutrino candidates}

\section{Time distribution}

Figure 9 shows the cumulative number of neutrino events as well as the cumulative number of event triggers plotted versus the day number in 1997. One can observe that the neutrino events follow the number of triggers, albeit with a small deficit during the Antarctic winter. This deficit is consistent with statistical fluctuations. (Actually, seasonal variations slightly decrease the downward muon rate during the Antarctic winter [28] and should result in a $10 \%$ deficit of triggers with respect to upward neutrino events.)

TABLE II. The cut efficiencies for the atmospheric neutrino Monte Carlo (MC) prediction, the atmospheric muon background Monte Carlo prediction, and the experimental data for 130 days of detector lifetime. Efficiencies are given for filter levels L1 to L4. L4 is the final selection. All errors are purely statistical. The final background prediction of 7 events has been normalized at trigger level.

\begin{tabular}{lcccc}
\hline \hline Filter level & $\begin{array}{c}\text { Atm. } \nu \\
\text { MC }\end{array}$ & $\begin{array}{c}\text { Atm. } \nu \text { MC } \\
\text { ANGSENS }\end{array}$ & $\begin{array}{c}\text { Atm. } \mu \text { MC } \\
(\text { Background })\end{array}$ & $\begin{array}{c}\text { Experimental } \\
\text { data }\end{array}$ \\
\hline Events at trigger level & 8978 & 5759 & $9.03 \times 10^{8}$ & $1.05 \times 10^{9}$ \\
Efficiency at level 1 & 0.34 & 0.37 & $0.4 \times 10^{-1}$ & $0.5 \times 10^{-1}$ \\
Efficiency at level 2 & 0.15 & 0.15 & $0.4 \times 10^{-3}$ & $0.4 \times 10^{-3}$ \\
Efficiency at level 3 & $0.7 \times 10^{-1}$ & $0.7 \times 10^{-1}$ & $0.7 \times 10^{-5}$ & $0.1 \times 10^{-4}$ \\
Efficiency after final cuts & $0.4 \times 10^{-1}$ & $0.4 \times 10^{-1}$ & $0.6 \times 10^{-8}$ & $0.2 \times 10^{-6}$ \\
No. of events & $362 \pm 4$ & $237 \pm 6$ & $7 \pm 5$ & 223 \\
passing final cuts & & & normalized & \\
\hline \hline
\end{tabular}




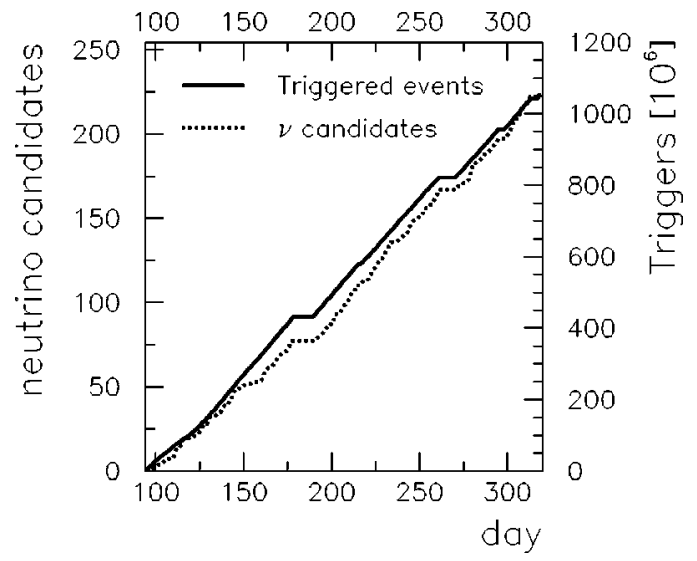

FIG. 9. The integrated exposure of the AMANDA detector in 1997. The figure shows the cumulative number of triggers (upper curve) and the number of observed neutrino events (lower curve) versus the day number. The intervals with zero gradient correspond to periods where the detector was not operating stably; data from these periods were excluded from the analysis.

\section{Zenith angle distribution}

Figure 10 shows the zenith angle distribution of the 223 neutrino candidates compared to the Monte Carlo prediction for atmospheric neutrinos [17] and the few remaining events predicted by background simulations. Note that the Monte Carlo prediction is normalized to experiment. (The total number of events is 362 for the atmospheric neutrino simulation and 223 for experiment, i.e., there is a deficit of 39 percent in the absolute number of events.) There is good agreement between the prediction and the experiment in the shape of the angular distribution.

\section{Characteristic distributions and visual inspection}

Four methods were used to evaluate the effectiveness of the analysis and the level of residual backgrounds: (a) $N$

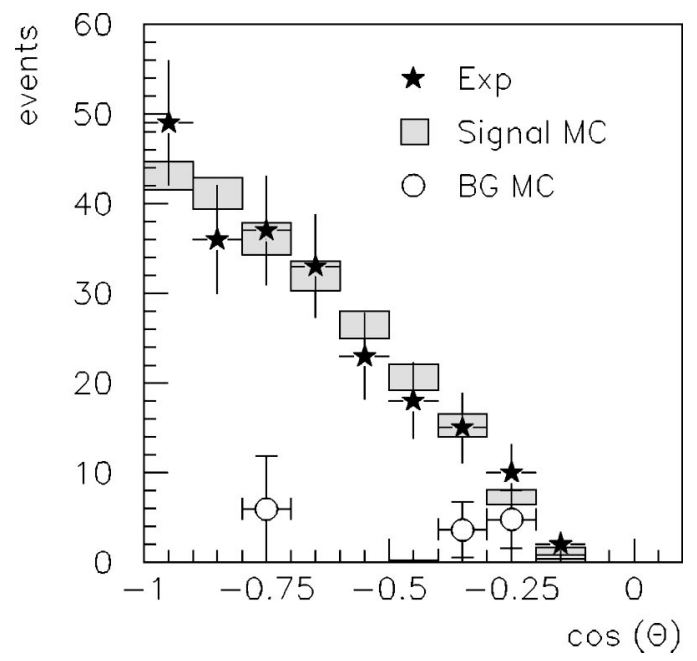

FIG. 10. Zenith angle distribution of the experimental data compared to simulated atmospheric neutrinos and a simulated background of downgoing muons produced by cosmic rays. In this figure the Monte Carlo prediction is normalized to the experimental data. The error bars report only statistical errors.
-1 cuts, (b) unbiased variables, (c) low level distributions, and (d) visual inspection.

(a) The $N-1$ test evaluates the $N$ final cuts one by one and yields an estimate of the background contamination in the final sample. One applies all but one of the final cuts (the one in the selected variable), and plots the data in this variable. In the signal region of this variable (defined by the later applied cut) shapes of experiment and signal Monte Carlo program should agree. In the background region, the experimental data should approach the expected background shape. Figure 11 shows four of these distributions. The applied cut is shown by a dotted line. All four cuts satisfy the test: the shape of the distributions agree reasonably well on both sides of the applied cuts. Two $N-1$ distribution from analysis II are shown in Fig. 19.

(b) An obvious test is the investigation of distributions of unbiased variables (i.e., variables to which no cuts have been applied) in the final neutrino sample. Here, the experimental distributions follow the Monte Carlo signal expectations nicely. Some deviations are observed, especially in the number of OMs hit and the velocity $v_{\text {lf }}$ obtained from the line fit (see Sec. IV A). However, as can be seen from Fig. 20 , part of these disagreements disappear if the standard atmospheric neutrino MC program is replaced by the ANGSENS $\mathrm{MC}$ version.

(c) In order to account for possible pathological low level features in the data sample (especially cross-talk), we (i) investigated basic pulse amplitude and pulse width (TOT) distributions and (ii) re-fitted all events after the cross-talk hit cleaning procedure applied in analysis II (which is tighter than the standard cross-talk cleaning introduced in Sec. IV A). Both these distributions and that for the recalculated zenith angles show no significant deviation from the previous ones. No cross-talk features are found in the resulting neutrino sample.

(d) Finally, a visual inspection of the full neutrino sample was performed, by visually displaying each event like in Fig. 4. The visual inspection gives consistent results with the other methods of background estimation and yields an upper limit on the background contamination of muons from random coincident air showers (see below).

\section{E. Background estimation}

The results of four independent methods of background estimation are summarized in Table III.

First, the background Monte Carlo program itself gives an estimate. It yields 7 events if rates are normalized to the trigger level (see Table II). Because the passing rates differ slightly between the experiment (higher) and the background Monte Carlo program (lower), we made the conservative choice to renormalize the background Monte Carlo program to the level 3 experimental passing rate. This gives an estimate of about 16 background events in our final sample.

From the $N-1$ distributions we obtained an alternative approximation of the residual background. We re-normalized both signal and background MC events in the background region to fit the number of experimental events in the background region. The number of re-normalized background 

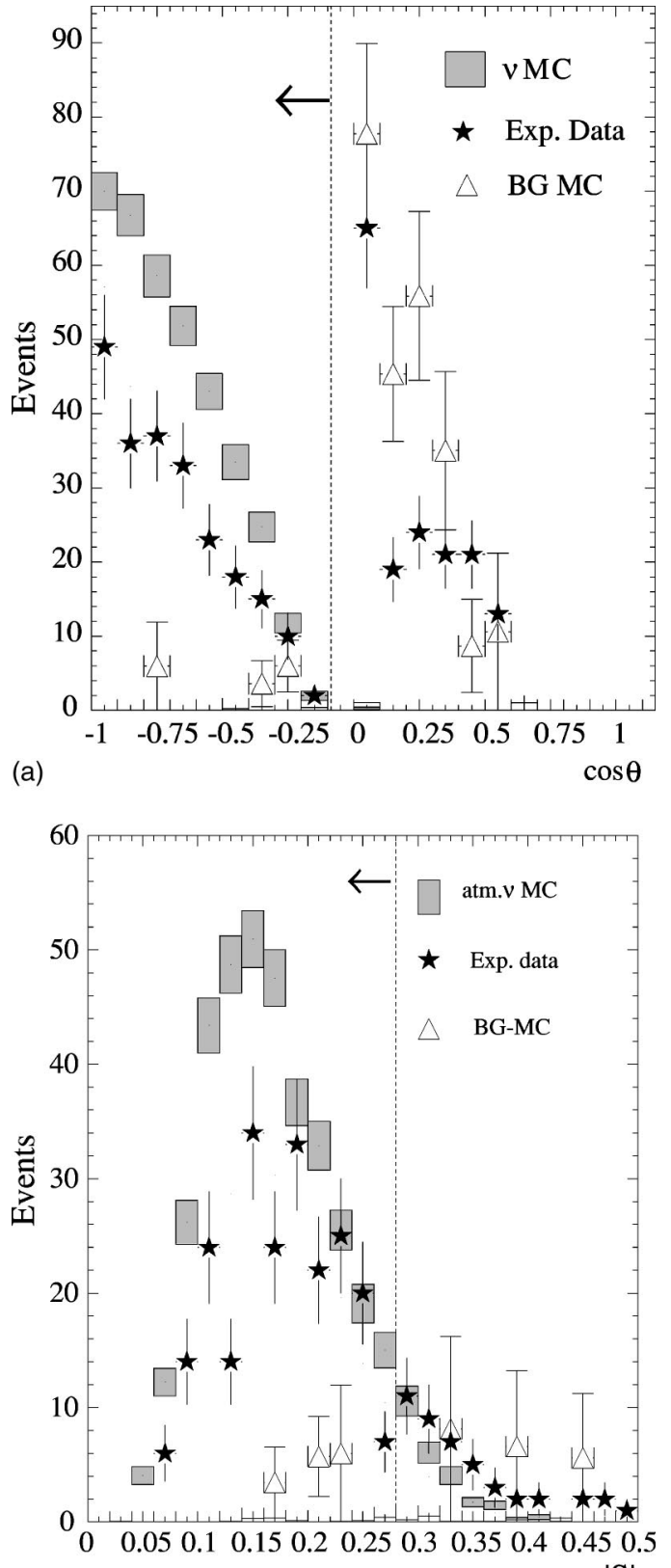

(b)

$|\mathrm{S}|$

FIG. 11. Two distributions of variables used as cut parameters in the last filter level (see Table I for an explanation of the variables). In both cases, all final cuts with the exception of the variable plotted have been applied. The cuts on the displayed parameters are indicated by the dashed vertical lines. Arrows indicate the accepted parameter space.

$\mathrm{MC}$ events in the signal region is then a background estimate. This estimate was performed $N$ times (once for each $N-1$ distribution). The average over all $N$ estimations yields 14 background events. Note that this averaging procedure is reasonable only for the case of independent cuts. With the method by which we have chosen the cut parameters, this condition is satisfied to first approximation.

We have found that cross-talk hits are related to the characteristic triple-peak structure in the distribution of the vertical component of the center of gravity of hits $\left(z_{\mathrm{COG}}\right)$ which
TABLE III. Various estimates of the background remaining in the experimental data sample of 223 neutrino candidates.

\begin{tabular}{lc}
\hline \hline BG estimation method & Estimation \\
\hline BG MC & $16 \pm 8$ \\
$N-1$ cuts & $14 \pm 4$ \\
$z_{\text {COG distributions }}$ & $<35$ \\
Visual inspection & $<23$ \\
\hline
\end{tabular}

has been discussed in Sec. V A-see Fig. 7 and also Fig. 14 (top). Since there are remaining cross talk hits which have survived the standard cleaning (see Sec. IV A), this distribution was studied in detail. As shown in Fig. 12, the final experimental sample of neutrino candidates shows no statistically significant excess with respect to the atmospheric neutrino Monte Carlo prediction in the regions of the characteristic peaks. Therefore, an upper limit on this special class of background was derived and yields $<35$ events.

The visual inspection of the neutrino sample yields 13 events. Seven of them show the signature of coincident muons from independent air showers; i.e., two well separated spatial concentrations of hits, each with a downward time flow but with the lower group appearing earlier than the upper one. Taking into account the scanning efficiencies which were determined by scanning signal and background Monte Carlo events, an upper limit of 23 events is obtained from visual inspection.

Combining the results from the above methods, the expected background is estimated to amount to 4 to $10 \%$ of the 223 experimental events.

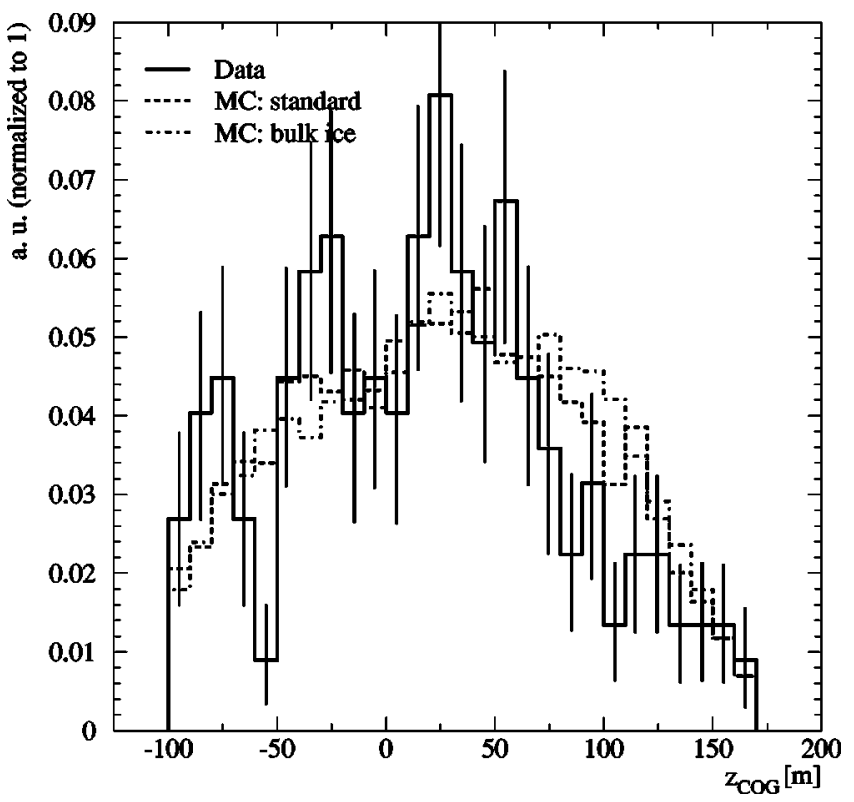

FIG. 12. Distributions of $z_{\mathrm{COG}}$ for the experiment and atmospheric neutrino signal Monte Carlo program $M C$ standard and $M C$ bulk ice denote two different ice models. The first includes vertical ice layers in accordance with Fig. 2; the second uses homogeneous ice. 


\section{ANALYSIS II}

The second analysis follows a different approach; instead of optimizing cuts to reject misreconstructed cosmic ray muons, this analysis concentrates on improving the reconstruction algorithm with respect to background rejection. The large downgoing muon flux implies that even a small fraction of downgoing muons misreconstructed as upgoing will produce a very large background rate. Equivalently, for each apparently upgoing event, there were many more downgoing muons passing the detector than there were upgoing muons; even though any single downgoing muon had only a small probability of faking an upgoing event, the total probability that the event was a fake is quite high.

\section{A. Bayesian reconstruction}

This analysis of the problem motivates a Bayesian approach [24] to event reconstruction. Bayes' theorem in probability theory states that for two assertions $A$ and $B$,

$$
P(A \mid B) P(B)=P(B \mid A) P(A),
$$

where $P(A \mid B)$ is the probability of assertion $A$ given that $B$ is true. Identifying $A$ with a particular muon track hypothesis $\mu$ and $B$ with the data recorded for an event in the detector, we have

$$
P(\mu \mid \text { data })=\mathcal{L}_{\text {time }}(\text { data } \mid \mu) P(\mu),
$$

where we have dropped a normalization factor $P$ (data) which is a constant for the observed event. The function $\mathcal{L}_{\text {time }}$ is the regular likelihood function of Eq. (1), and $P(\mu)$ is the so-called prior function, the probability of a muon $\mu$ $=\mu(x, y, z, \theta, \phi)$ passing through the detector.

For this analysis, we have used a simple one-dimensional prior function, containing the zenith angle information at trigger level in Fig. 3. By accounting in the reconstruction for the fact that the flux of downgoing muons from cosmic rays is many orders of magnitude larger than that of upgoing neutrino-induced muons, the number of downgoing muons that are misreconstructed as upgoing is greatly reduced. It should be noted that the objections that are often raised with respect to the use of Bayesian statistics in physics are not relevant to this problem: the prior function is well defined and normalized and independently known to relatively good precision, consisting only of the fluxes of cosmic ray muons and atmospheric muon neutrinos.

\section{B. Removal of instrumental artifacts}

The Bayesian reconstruction algorithm is highly efficient at rejecting downgoing muon events. Of $2.6 \times 10^{8}$ events passing the fast filter, only $5.8 \times 10^{4}$ are reconstructed as upgoing. By contrast, the standard maximum likelihood reconstruction produces about $2.4 \times 10^{7}$ false upgoing reconstructions. However, less than a thousand neutrino events are predicted by Monte Carlo program, so it is clear that a significant number of misreconstructions remain.

Detailed inspection of the $5.8 \times 10^{4}$ events reveals that the vast majority is produced by cross-talk overlaid on triggers


FIG. 13. Pulse amplitude vs duration for modules on the outer strings. Normal hits lie in the distribution shown in the upper figure. High amplitude pulses of more than a few photoelectrons are valid only if the pulse width is also large. Cross-talk induced pulses of high amplitude are characterized by small time over threshold (TOT). The cutoff seen at high amplitude is due to saturation of the amplitude readout electronics.

from downgoing muons emitting bright stochastic light near the detector. This cross-talk confuses the reconstruction algorithm, producing apparently upgoing tracks. Because cross-talk is not included in the detector simulation, the characteristics of the fakes are not predicted well by the simulation, and the rate of misreconstruction is much higher than predicted.

The cross-talk is removed by additional hit cleaning routines developed by examination of this cross-talk enriched data set. For example, cross-talk in many channels can be identified in scatter plots of pulse width vs amplitude, as shown in Fig. 13. The pulse width is measured as time over threshold (TOT). Real hits form the distribution shown on the left. High amplitude pulses should have large pulse width. This is not the case for cross-talk induced pulses. In channels with high levels of cross-talk, an additional vertical 

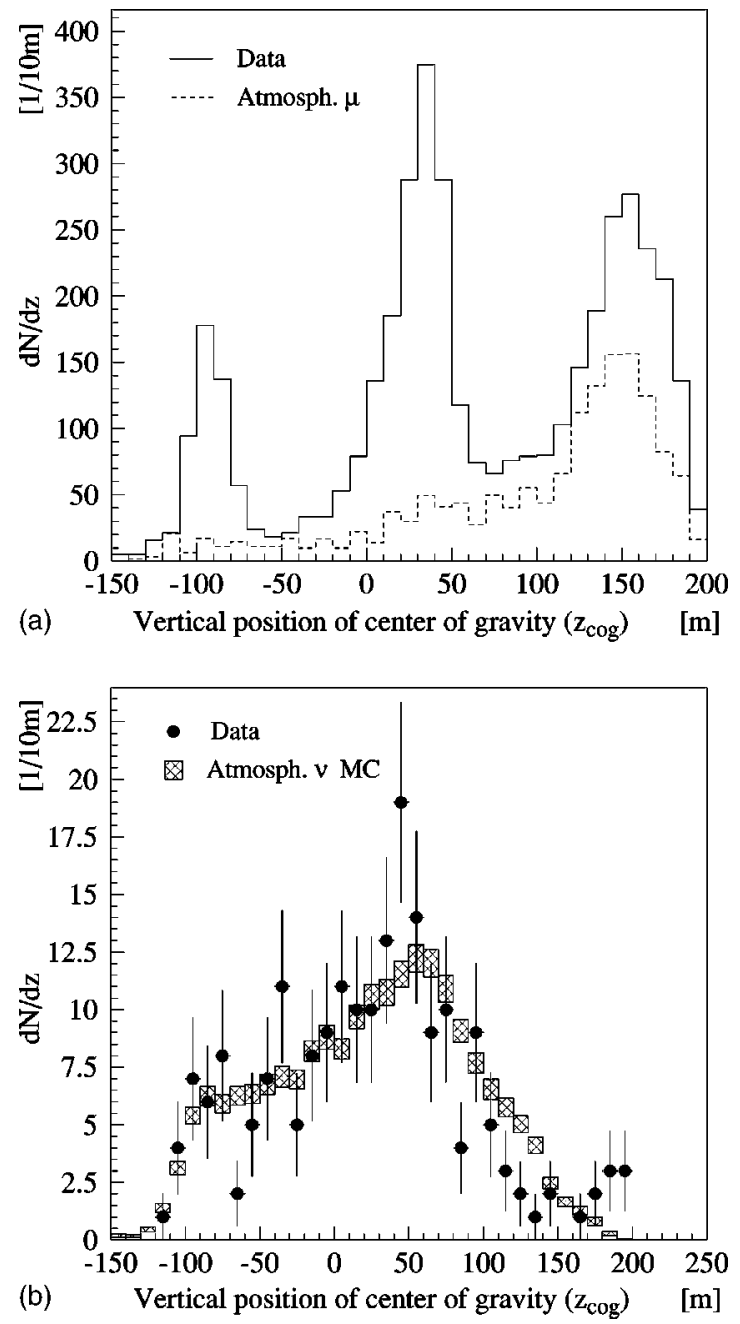

FIG. 14. Top: Event center of gravity distribution after reconstruction with special cross-talk cleaning algorithms applied to the events. Unsimulated background remains. Bottom: The data agree with the neutrino signal after application of additional quality cuts.

band is found at high amplitudes but short pulse widths, as seen in the lower figure.

Other hit cleaning algorithms use the time correlation and amplitude relationship between real and cross-talk pulses and a map of channels susceptible to cross-talk and the channels to which they are coupled. An additional instrumental effect, believed to be caused by fluctuating high voltage levels, produces triggers with signals from most OMs on the outer strings but none on the inner four strings; some 500 of these bogus triggers were also removed from the data set. The $5.8 \times 10^{4}$ upgoing events were again reconstructed after the additional hit cleaning was applied. Only $4.9 \times 10^{3}(8.4 \%)$ of the events remained upgoing, compared to an expectation from Monte Carlo program of 1855 atmospheric muon events $(37.8 \%$ of the total before the additional cleaning), and 555 atmospheric neutrino events. Figure 14 (top) shows that while there has been a significant reduction in the instrumental backgrounds, an unsimulated structure still remains in the center-of-gravity (COG) distribution for these remaining data events. The application of additional quality criteria

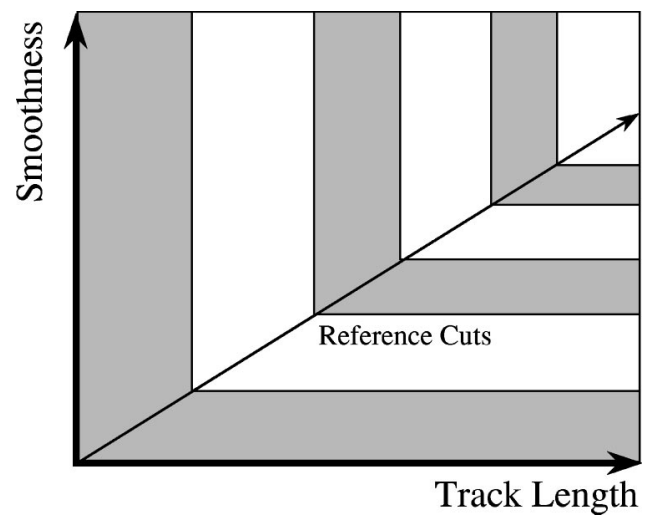

FIG. 15. Definition of event quality. Events are plotted in $\mathrm{N}$-dimensional cut space (two dimensions are shown here for clarity). A line is drawn from the origin (no cuts) through a selected set of cuts, and the space is divided into rectangular shells of equal width. Events are assigned a quality $q$ according to the shell in which they are found.

brings this distribution in agreement, as shown in Fig. 14 (bottom).

\section{Quality cuts}

The improvements in the reliability of the reconstruction algorithm described above obviated the need for large numbers of cut parameters or for careful optimization of the cuts. Because the signal-to-noise ratio of the upward-reconstructed data is quite high to begin with, we have the possibility of comparing the behavior of real and simulated data over a wide range of cut strengths to verify that the data agree with the predictions for upgoing neutrino-induced muons, not only in number but also in their characteristics. Using the cut parameters described in Sec. IV C (with the likelihood replaced by the Bayesian posterior probability) and a requirement that events fitted as relatively horizontal by the line fit filtering algorithm not be reconstructed as steeply upgoing by the full reconstruction (a requirement that suppresses residual cross-talk misreconstructions), an index of event quality was formed.

To do so, we rescale the six quality parameters described above by the cumulative distributions of the simulated atmospheric neutrino signal, and consider the six-dimensional cut space formed by the rescaled parameters. A point in this space corresponds to fixed values of the quality parameters, and events can be assigned to locations based on their track length, sphericity, and so forth.

It is difficult to compare the distributions of data and simulated up- and downgoing muons directly because of the high dimensionality of the space. We therefore project the space down to a single_ " "quality" — dimension by dividing it into concentric rectangular shells, as illustrated in Fig. 15. The vertex of each shell lies on a line from the origin through a reference set of cuts which are believed to isolate a fairly pure set of neutrino events. Events in the full cut space are assigned an overall quality value, based on the shell in which they lie. 


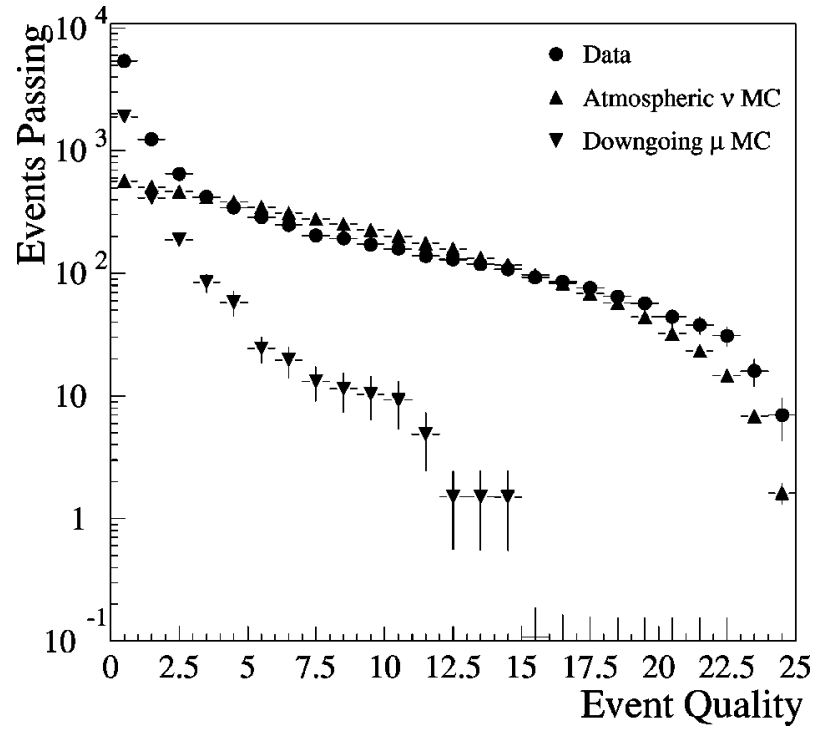

FIG. 16. Numbers of events above a certain quality level, for downgoing muon Monte Carlo simulations, atmospheric neutrino Monte Carlo simulations, and experimental data.

With this formulation we can compare the characteristics of the data to simulated neutrino and cosmic-ray muon events. Figure 16 compares the number of events passing various levels of cuts; i.e., the integral number of events above a given quality. At low qualities, $q \leqslant 3$, the data set is dominated by misreconstructed downgoing muons, data as well as the simulated background exceed the predicted neutrino signal. At higher qualities, the passing rates of data closely track the simulated neutrino events, and the predicted background contamination is very low.

We can investigate the agreement between data and Monte Carlo simulations more systematically by comparing the differential number of events within individual shells, rather than the total number of events passing various levels of cuts. This is done in Fig. 17, where the ratios of the number of events observed to those predicted from the combined signal and background simulations are shown. One can see that at low quality levels there is an excess in the number of misreconstructed events observed. This is mainly due to remaining cross-talk. There is also an excess, though statistically less significant, at very high quality levels, which is believed to be caused by slight inaccuracies in the description of the optical parameters of the ice. Nevertheless, over the bulk of the range there is close agreement between the data and the simulation, apart from an overall normalization factor of 0.58 . The absolute agreement is consistent with the systematic uncertainties. It should be emphasized that the quality parameter is a convolution of all six quality parameters, and so the flat line in Fig. 17 demonstrates agreement in the correlations between cut parameters.

\section{Background estimation and signal description}

If we reduce the 4917 upward-reconstructed events by requiring a quality of at least 7 on the scale of Fig. 16, we obtain a set of 204 neutrino candidates. The background contamination, which is due to misreconstructed downgoing

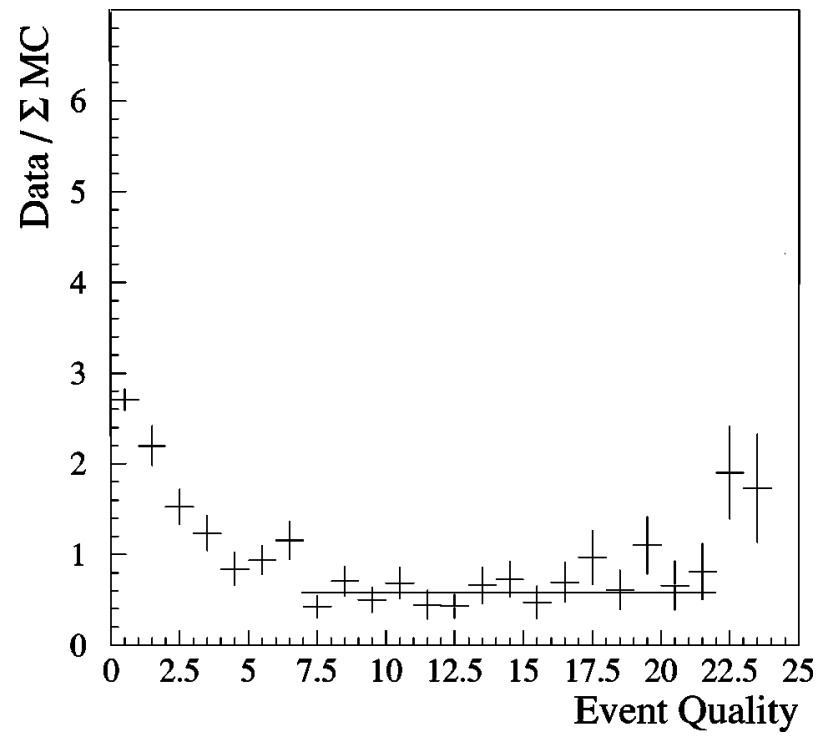

FIG. 17. Ratio of data to Monte Carlo simulations (cosmic ray muons plus atmospheric neutrinos). Unlike Fig. 16, the plot is differential - the ratio at a particular quality does not include events at higher or lower qualities.

muons, was estimated in three ways. The first way is to simulate the downgoing muon flux, bearing in mind that we are looking at a very low tail $\left(10^{-8}\right)$ of the total muon distribution. The second way is to renormalize the signal simulation by the factor of 0.58 obtained from Fig. 17 and subtract the predicted events from the observed data set (accepting the excess at extremely high qualities, however, as signal). The third way, a cross check on the first two methods, is to examine the data looking for fakes due to unsimulated effects such as cross-talk, independent coincident downgoing muons, and so forth. All three methods yield estimates of $5-10 \%$ contamination.

The zenith angle distribution for the 204 events is shown in Fig. 18, and compared to that for the simulation of atmospheric neutrinos. In the figure the Monte Carlo events are normalized to the number of observed events to facilitate comparison of the shapes of the distributions. The agreement in absolute number is consistent with the systematic uncertainties in the absolute sensitivity and the flux of high energy atmospheric neutrinos. The shape of the distribution of data is statistically consistent with the prediction from atmospheric neutrinos. Figure 14 (bottom) shows the distribution of the $z_{\mathrm{COG}}$ parameter for the 204 events. The level 7 quality cuts have removed the remainder of the instrumental events left after the Bayesian reconstruction with the improved cross-talk cleaning algorithm, bringing the data events in line with the atmospheric neutrino expectations. The efficiencies corresponding to the three steps of the data analysis: (1) events reconstructed upward, (2) events reconstructed upward with cross-talk cleaning, and (3) with additional level 7 quality cuts are summarized in Table IV.

Figure 19 (top) shows the smoothness distribution for events that have passed the quality level 7 cuts for the five observables except smoothness. The vertical dashed line at smoothness $\sim 0.29$ shows the value of the level 7 smooth- 


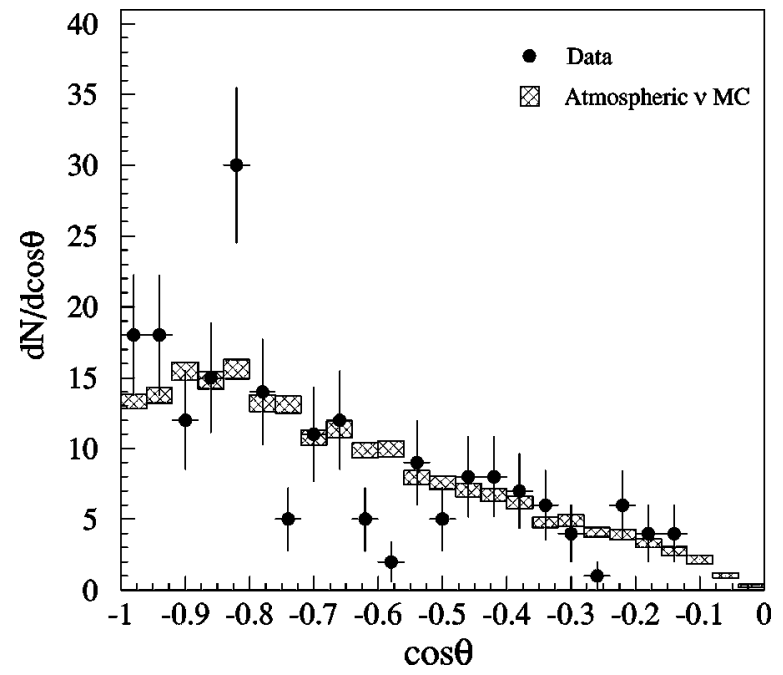

FIG. 18. The zenith angle distribution of upward reconstructed events. The size of the hatched boxes indicates the statistical precision of the atmospheric neutrino simulation. The Monte Carlo prediction is normalized to the data.

ness cut. This cut removes the tail of fake events leaving a good agreement between remaining data and the Monte Carlo expectation. Figure 19 (bottom) shows the same plot for the direct length variable. Again, a clear tail of fake events is removed by requiring a direct length of greater than $70 \mathrm{~m}$.

\section{SYSTEMATIC UNCERTAINTIES}

As a novel instrument, AMANDA poses a unique challenge of calibration. There are no known natural sources of high energy neutrinos, apart from atmospheric neutrinos, whose observation could be used to measure the detector's response. Understanding the behavior of the detector is thus a difficult task, dependent partly on laboratory measurements of the individual components, partly on observations of artificial light sources embedded in the ice, and partly on observations of downgoing muons. Even with these measurements, uncertainties in various properties that systematically affect the response of the detector persist, which prevent us at this time from making a precise measurement of the atmospheric neutrino flux. The primary sources of systematic uncertainties, and their approximate effects on the number of upgoing atmospheric neutrinos in the final data sample, as
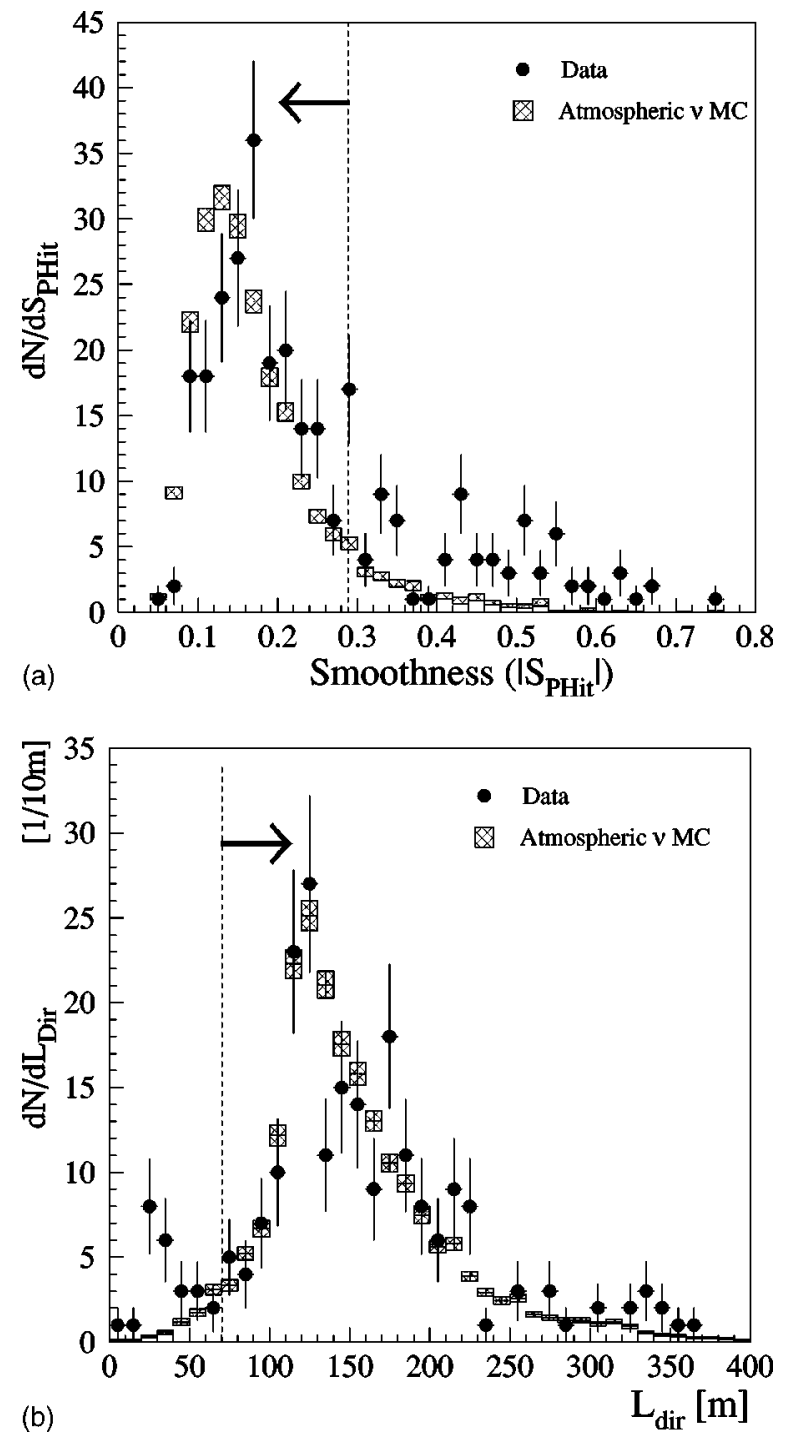

FIG. 19. Smoothness and direct length variables where quality level 7 cuts have been applied in all but the displayed variable $(N$ -1 cuts; see also Sec. V D 3, Fig. 11). The vertical dashed lines with the arrow indicate the region of acceptance in the displayed variable. In each case, a clear tail of fake events is removed by application of the cut, leaving good agreement in shape between the remaining events and the Monte Carlo expectation.

TABLE IV. Event numbers for experimental data and Monte Carlo simulations for four major stages in the analysis. The errors quoted are statistical only.

\begin{tabular}{|c|c|c|c|}
\hline & $\begin{array}{l}\text { Monte Carlo } \\
\text { downgoing } \mu\end{array}$ & $\begin{array}{l}\text { Monte Carlo } \\
\text { atmospheric } \nu\end{array}$ & Data \\
\hline Events triggered & $8.8 \times 10^{8}$ & 8978 & $1.05 \times 10^{9}$ \\
\hline Efficiency: Reconstructed upgoing & $0.55 \times 10^{-5}$ & & $0.55 \times 10^{-4}$ \\
\hline $\begin{array}{l}\text { Efficiency: Reconstructed upgoing } \\
\text { (with cross-talk cleaning) }\end{array}$ & $(2.1 \pm 0.08) \times 10^{-6}$ & $(6.2 \pm 0.06) \times 10^{-2}$ & $4.7 \times 10^{-6}$ \\
\hline Efficiency: Final cuts $(q \geqslant 7)$ & $(1.9 \pm 0.6) \times 10^{-8}$ & $(3.1 \pm 0.03) \times 10^{-2}$ & $1.9 \times 10^{-7}$ \\
\hline No. of events: Quality $\geqslant 7$ & $17 \pm 5$ & $279 \pm 3$ & 204 \\
\hline
\end{tabular}



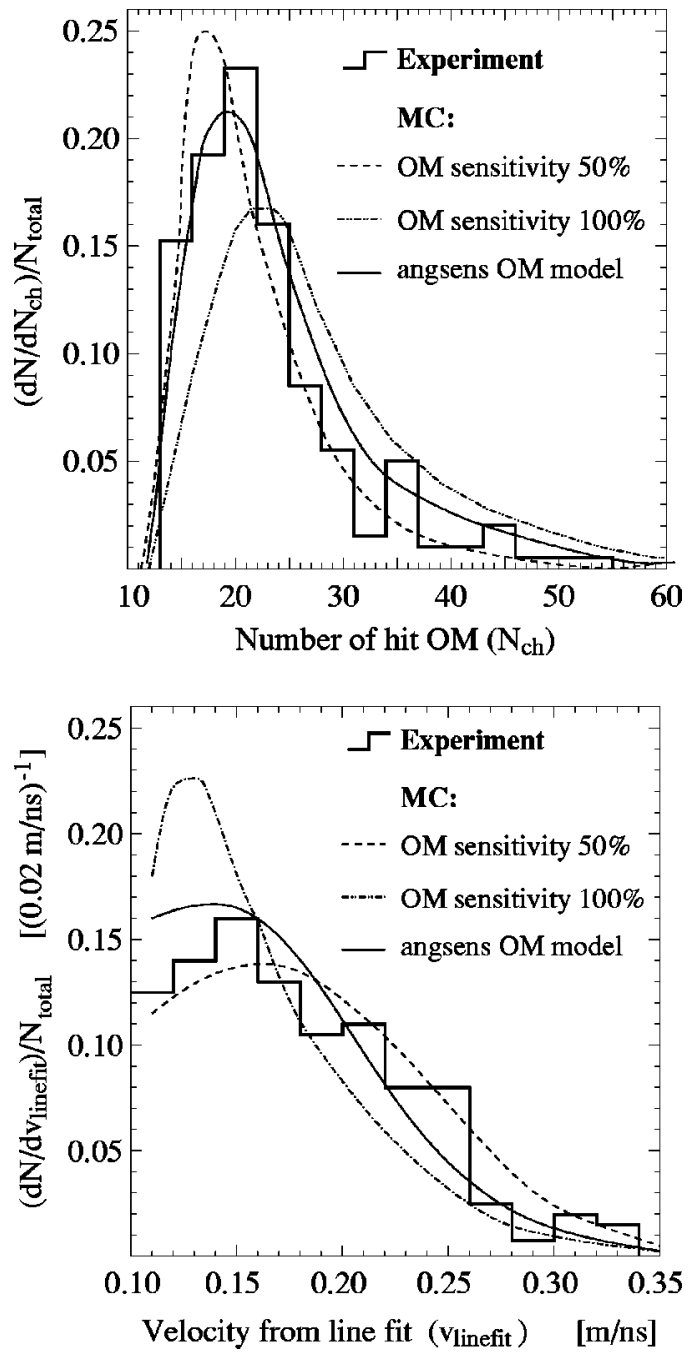

FIG. 20. Distributions of two variables that are affected by the OM sensitivity, comparing different signal Monte Carlos events to the observed data. Top: the number of OMs hit $\left(N_{\mathrm{ch}}\right)$; bottom: the event velocity for a simplified fit (line fit, $\left.v_{\text {linefit }}\right)$.

determined by variation of the simulations, are listed below.

As discusssed in Secs. II and III, AMANDA is embedded in a natural medium, which is the result of millennia of climatological history, that has left its mark in the form of layers of particulate matter affecting the optical properties of the ice. Furthermore, the deployment of optical modules requires the melting and refreezing of columns of the ice. This cycle results in the formation of bubbles in the vicinity of the modules, which increase scattering and affect the sensitivity of the optical modules in ways that are not yet fully understood. The effects of this local hole ice are difficult to separate from the intrinsic sensitivity of the OMs. The uncertainties in the neutrino rate are approximately $15 \%$ from the bulk ice layer modeling in the Monte Carlo simulation, and as much as $50 \%$ from the combined effects of the properties of the refrozen hole ice close to the OMs, and the intrinsic OM sensitivity, and angular response.

Figure 20 shows two variables that are sensitive to the absolute OM sensitivity: the number of OMs hit and the velocity of the line fit. The systematic effects of varying OM

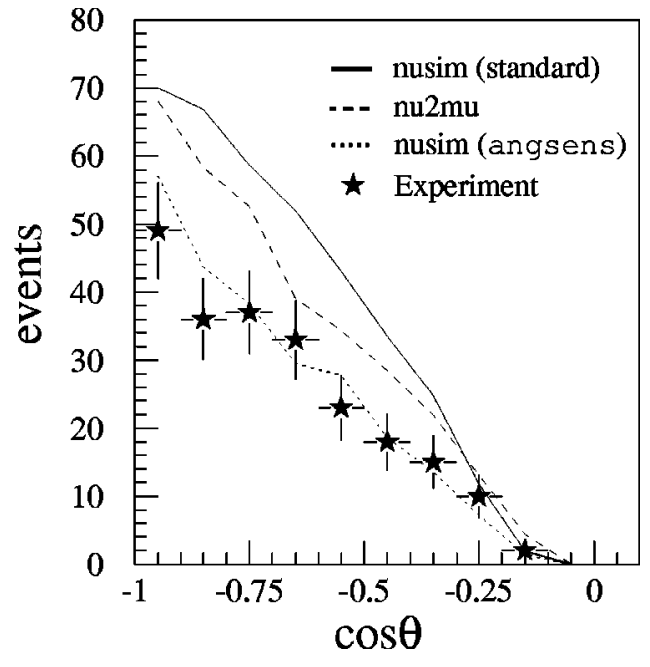

FIG. 21. Distribution of simulated zenith angles for different neutrino generators and also for a modified angular sensitivity of the OM.

sensitivity on the hit multiplicity for analysis I are shown on the top. The peak of the multiplicity distribution for the standard Monte Carlo events (nominal efficiency 100\%-dashed line) lies at a higher value than for the data. Reducing the simulated OM sensitivity by $50 \%$ results in a peak at lower values than the data. The other variable strongly affected by the OM sensitivity - the velocity of the line fit, introduced in Sec. IV A) - is the apparent velocity of the observed light front traveling through the ice; see Fig. 20 (bottom).

As a next step, we investigated the effect of the ANGSENS OM model (first introduced in Sec. III) on the atmospheric neutrino Monte Carlo simulation. The results of this simulation gave a more consistent description of the experiment for several variables-e.g., the hit multiplicity (the dotted line in Fig. 20) — and they produced the absolute neutrino event prediction closer to what was found in Analysis I (236.9 events predicted, 223 observed). Similar effects are seen when this Monte Carlo simulation is used with analysis II, however the number of predicted events is $25 \%$ smaller than observed. Thus the ANGSENS model, while encouraging, does not completely predict the properties of observed events in both analyses.

Another uncertainty lies in the Monte Carlo routines used to propagate muons through the ice and rock surrounding the detector. A comparison of codes based on [9] and [11] indicates that different propagators may change the event rates by some $25 \%$.

Other factors include the simulation of the data acquisition electronics and possible errors in the time calibrations of individual modules. These effects have been studied by systematically varying relevant parameters in the Monte Carlo simulations. For realistic levels of variation, these effects are well below the $10 \%$ level.

Figure 21 demonstrates how the zenith angle distribution depends on different atmospheric neutrino event generators (our standard generator NUSIM [17] and another generator NU2MU [29]), and also on the chosen angular sensitivity of the optical module. Neutrino flavor oscillations lead to a fur- 


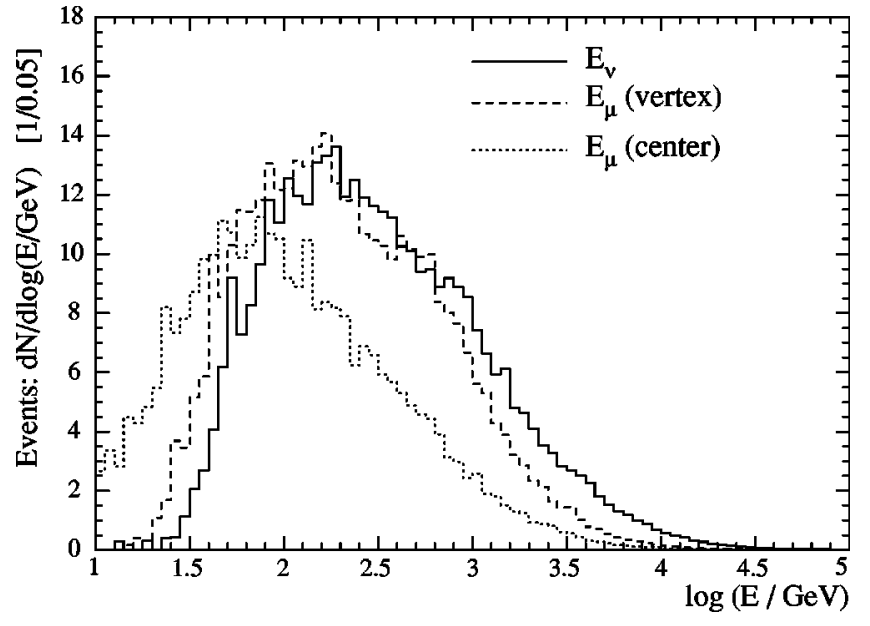

FIG. 22. Energy distributions for simulated atmospheric neutrino events which pass the final neutrino cuts. The effect of neutrino oscillations has not been taken into account. The figure shows the neutrino and muon energies at the interaction vertex and the energy of the muons at the point of closest approach to the detector center.

ther reduction of the ANGSENS prediction by $5.4 \%$ (in particular, close to the vertical direction), assuming $\sin ^{2} 2 \theta=1$ and $\Delta m^{2}=2.5 \times 10^{-3} \mathrm{eV}^{2}[30,38]$. The prediction is reduced by $11 \%$ if the largest allowed $\Delta m^{2}$ is used.

The combined effect of all these systematic uncertainties is sufficiently large that simulations of a given atmospheric neutrino flux can produce predictions for the event rate varying by a factor of two. By contrast, the estimated theoretical uncertainty in the atmospheric neutrino flux, at the energies probed by these analyses, is 30\% [31]. The effect of neutrino oscillations with the Super-K preferred parameters would be less than $10 \%$ at these energies.

\section{SYNTHESIS AND GENERAL OVERVIEW}

Both analyses I and II are able to separate more than 200 neutrino event candidates from the 130.1 days of AMANDAB10 detector lifetime in 1997. Based on atmospheric neutrino simulations we find that about $4 \%$ of the total number of events triggered by upward moving neutrinos passed the final selection. A total deficit in the event rate of about 35\% with respect to the standard neutrino Monte Carlo prediction is found for both analyses. An event overlap of 102 experimental events is observed, consistent with a predicted overlap of $119 \pm 13$ from the atmospheric neutrino Monte Carlo prediction. Thus, the combined sample of data provides about 300 neutrino candidates. Both analyses estimate their residual background to be about $10 \%$ of the number of neutrino event candidates.

Figure 22 shows the energy distribution of the simulated neutrinos and the corresponding muon events. Ninety percent of all Monte Carlo signal events have muon (neutrino) energies between 48 (66) GeV and 1.8 (3.4) TeV. The dominant part of the signal events in this analysis comes from neutrino energies below $1 \mathrm{TeV}$. Figure 23 shows the effective area as a function of the zenith angle for two ranges of the

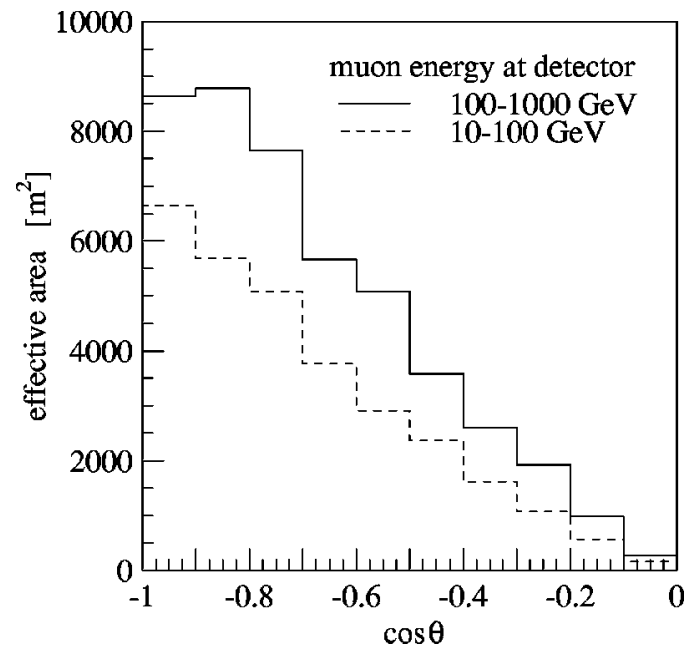

FIG. 23. Effective area for muons versus zenith angle. The energy of the muons is given at the point of closest approach to the detector.

muon energy at the point of closest (POC) approach to the detector. The effective area for muons with energies at POC between 100 and $1000 \mathrm{GeV}$ is $3.9 \times 10^{4} \mathrm{~m}^{2}$ at trigger level and $2800 \mathrm{~m}^{2}$ after application of the neutrino selection cuts. It should be noted that much higher effective areas are possible when searching for neutrinos from astrophysical point sources [32] or from gamma ray bursts [33].

Figure 24 shows the point spread function of the reconstructed muon trajectory with respect to the true muon direction. Based on Monte Carlo simulations, we find a median angular resolution of muons from atmospheric neutrinos of $3.2^{\circ}$ for the final sample. A more detailed study of the angular resolution can be found in [25,34,35]. Figure 25 shows the skyplot (equatorial coordinates) of all the candidate neutrino events found across both analyses. The distribution of the events on the skyplot is consistent with a random distribution.

\section{CONCLUSIONS}

The AMANDA-B10 data from 130.1 days of livetime during the austral winter of 1997 have been analyzed in an

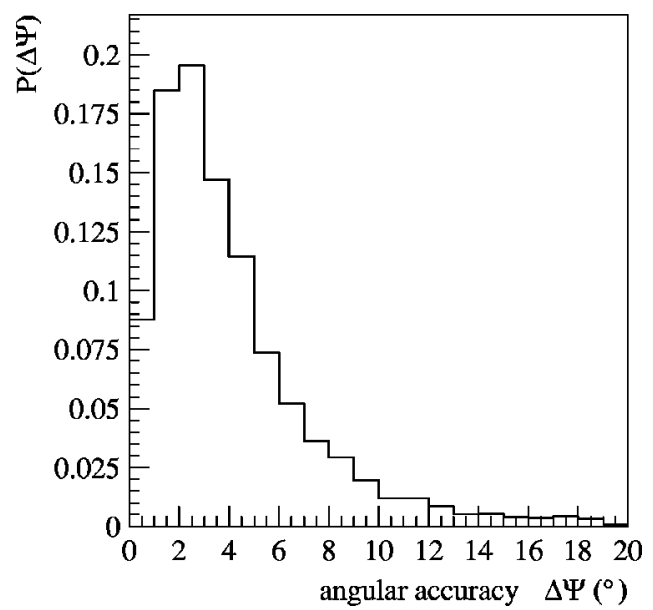

FIG. 24. Monte Carlo simulation of the angular resolution for muons that pass the final selection criteria. The median error is $3.2^{\circ}$. 


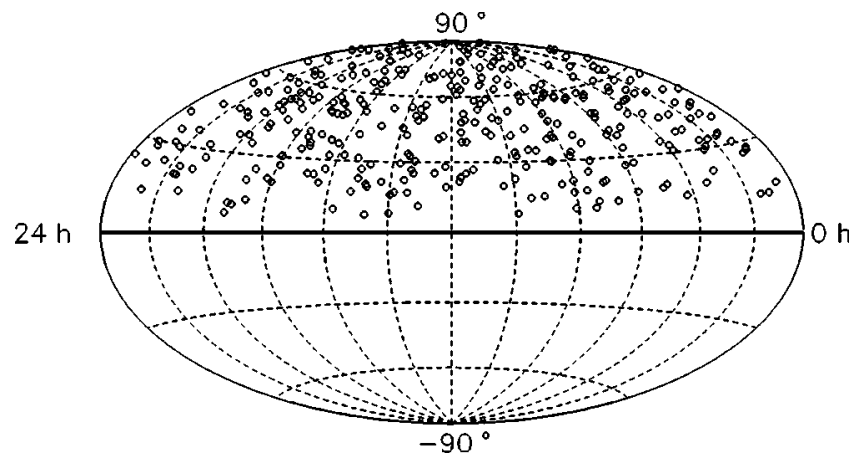

FIG. 25. Neutrino skyplot of upgoing events as seen with AMANDA-B10 in 1997 in equatorial coordinates. In this figure, neutrino events from both analyses are combined. The background of non-neutrino events is estimated to be less than $10 \%$.

effort to detect high energy atmospheric neutrino events, and to compare their properties to expectations. Two working groups in the collaboration, using differing reconstruction, cut optimization and instrumental event rejection techniques, produced sets of 223 and 204 neutrino candidates, respectively. Several methods of background estimation put the residual event contamination from downgoing atmospheric muons and instrumental artifacts at about $10 \%$. Taking into account systematic uncertainties, the observed event numbers are consistent with systematically varied atmospheric neutrino Monte Carlo predictions, which are from 150-400 events. The range of these predictions is dominated by uncertainties in the neutrino flux, in the understanding of pho- ton propagation through the bulk ice and the refrozen hole ice, and in muon propagation and energy loss. The Monte Carlo prediction suggests that $90 \%$ of the selected events are produced by neutrinos in the energy range of $\sim 66 \mathrm{GeV}$ to 3.4 TeV. The observation of atmospheric neutrinos in line with expectations establishes AMANDA-B10 as a working neutrino telescope. We finally note that many of the procedures for signal separation simplify considerably in larger detectors. In particular, first results from AMANDA-II $[36,37]$ demonstrate that the neutrino signal is separated with much higher efficiency and with fewer cuts than for AMANDA-B10.

\section{ACKNOWLEDGMENTS}

This research was supported by the following agencies: U.S. National Science Foundation, Office of Polar Programs; U.S. National Science Foundation, Physics Division; University of Wisconsin Alumni Research Foundation; U.S. Department of Energy; Swedish Natural Science Research Council; Swedish Research Council; Swedish Polar Research Secretariat; Knut and Alice Wallenberg Foundation, Sweden; German Ministry for Education and Research; U.S. National Energy Research Scientific Computing Center (supported by the Office of Energy Research of the U.S. Department of Energy); UC-Irvine AENEAS Supercomputer Facility; Deutsche Forschungsgemeinschaft (DFG). D. F. Cowen acknowledges the support of the NSF CAREER program and C. Pérez de los Heros acknowledges support from the EU 4th framework of Training and Mobility of Researchers.
[1] E. Andrés et al., Astropart. Phys. 13, 1 (2000).

[2] E. Andrés et al., Nature (London) 410, 441 (2001).

[3] AMANDA Collaboration, K. Woschnagg, in Proceedings of the $26^{\text {th }}$ International Cosmic Ray Conference (IUPAP, Salt Lake City, UT, 1999), Vol. 2, pp. 200-203.

[4] P. B. Price, K. Woschnagg, and D. Chrikin, Geophys. Res. Lett. 27, 2129 (2000).

[5] J. Ahrens et al., Astropart. Phys. 16, 345 (2002).

[6] S. N. Boziev et al., INR Report P-0630 (unpublished).

[7] D. Heck et al., Report FZKA 6019, Forschungszentrum Karlsruhe, Karlsruhe, Germany (unpublished).

[8] D. Heck, in Simulation and Analysis Methods for Large Neutrino Telescopes (DESY, Zeuthen, Germany, 1998), pp. 228234, DESY-PROC-1999-01.

[9] W. Lohmann, R. Kopp, and R. Voss, Report CERN-85-03, Geneva, Switzerland (unpublished).

[10] R. Kopp, W. Lohmann, and R. Voss, MUDEDX: Parametrization and Monte Carlo generation of energy loss of high energy muons (1-10000 GeV); for astrophysicists up to $100000 \mathrm{GeV}$, Version 2.02, 1995 (private communication).

[11] P. Lipari and T. Stanev, Phys. Rev. D 44, 3543 (1991).

[12] W. Rhode and D. Chirkin, in Proceedings of the $27^{\text {th }}$ International Cosmic Ray Conference (Copernicus Gesellschaft, Hamburg, Germany, 2001), Vol. 3, pp. 1017-1020.

[13] B. Wiebel-Sooth and P. L. Biermann, Numerical Data and
Functional Relationships in Science and Technology, LandoltBörnstein, New Series, Vol. VI 3C (Springer-Verlag, Berlin, Heidelberg, 1998), Chap. 7.6, pp. 37-90.

[14] A. Karle, in Simulation and Analysis Methods for Large Neutrino Telescopes (Ref. [8]), pp. 174-185, DESY-PROC-1999-01.

[15] S. Hundertmark, Ph.D. thesis, Humboldt Universität zu Berlin, Berlin, Germany, 1999, available at URL http://amanda.berkeley.edu/manuscripts/

[16] S. Hundertmark, in Simulation and Analysis Methods for Large Neutrino Telescopes (Ref. [8]), pp. 276-286, DESY-PROC-199901.

[17] G. C. Hill, Astropart. Phys. 6, 215 (1997).

[18] G. C. Hill, Ph.D. thesis, University of Adelaide, Adelaide, Australia, 1996.

[19] P. Lipari, Astropart. Phys. 1, 195 (1993).

[20] J. Ahrens et al. (unpublished).

[21] V. Stenger, DUMAND Internal Report HDC-1-90.

[22] C. Wiebusch, in Simulation and Analysis Methods for Large Neutrino Telescopes (Ref. [8]), pp. 302-316, DESY-PROC-199901.

[23] D. Pandel, Diploma thesis, Humboldt-University, Berlin, 1996.

[24] G. C. Hill, in Proceedings of the $27^{\text {th }}$ International Cosmic Ray Conference (Ref. [12]), Vol. 3, pp. 1279-1282.

[25] A. Biron, Ph.D. thesis, Humboldt Universtität zu Berlin, Berlin, Germany, 2002, available at URL 
http://amanda.berkeley.edu/manuscripts/

[26] T. R. DeYoung, Ph.D. thesis, University of Wisconsin, Madison, 2001, available at URL http://amanda.berkeley.edu/manuscripts/

[27] M. Gaug, Diploma thesis, Humboldt Universität zu Berlin, Berlin, Germany, 2001, DESY-THESIS-2001-022, available at URL http://amanda.berkeley.edu/manuscripts/.

[28] AMANDA Collaboration, A. Bouchta, in Proceedings of the 26th International Cosmic Ray Conference, (Ref. [3]), HE 3.2.11.

[29] E. Dalberg, Ph.D. thesis, Stockholm University, Stockholm, Sweden, 1999, available at URL http://amanda.berkeley.edu/manuscripts/

[30] Super-Kamiokande Collaboration, J. Kameda, in Proceedings of the $27^{\text {th }}$ International Cosmic Ray Conference (Ref. [12]), Vol. 3, pp. 1057-1060.

[31] T. K. Gaisser, "Uncertainty in flux of Atmospheric Neutrinos: Implications for Upward Muons in Amanda B10," Internal
Report 20001201, 2000.

[32] J. Ahrens et al., "Search for point sources of high energy neutrinos with the AMANDA detector."

[33] AMANDA Collaboration, R. Hardtke and G. Barouch, in Proceedings of the $27^{\text {th }}$ International Cosmic Ray Conference (Ref. [12]), Vol. 3, pp. 1121-1124.

[34] AMANDA Collaboration, "Calibration and Survey of AMANDA with the SPASE detectors" (to be published).

[35] AMANDA Collaboration, G. Spiczak, in Proceedings of the $27^{\text {th }}$ International Cosmic Ray Conference (Ref. [12]), Vol. 3, pp. 977-980.

[36] AMANDA Collaboration, R. Wischnewski, in Proceedings of the $27^{\text {th }}$ International Cosmic Ray Conference (Ref. [12]), Vol. 3, pp. 1105-1108.

[37] AMANDA Collaboration, S. W. Barwick, in Proceedings of the $27^{\text {th }}$ International Cosmic Ray Conference (Ref. [12]), Vol. 3, pp. 1105-1108.

[38] S. Fukuda et al., Phys. Rev. Lett. 85, 3999 (2000). 